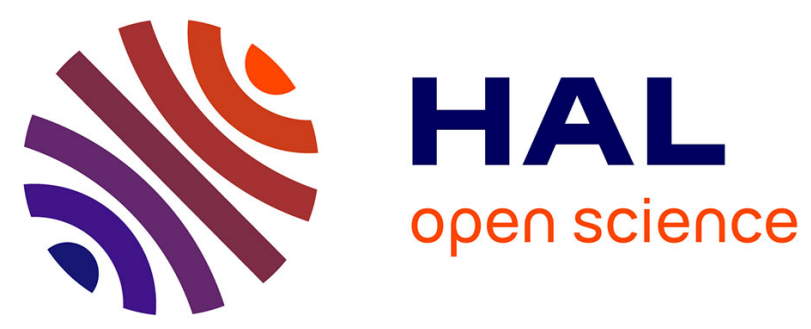

\title{
DUAL POLARIZED WIRELESS COMMUNICATIONS: FROM PROPAGATION MODELS TO SYSTEM PERFORMANCE EVALUATION
}

Claude Oestges, Bruno Clerkx, Maxime Guillaud, Merouane Debbah

\section{To cite this version:}

Claude Oestges, Bruno Clerkx, Maxime Guillaud, Merouane Debbah. DUAL POLARIZED WIRELESS COMMUNICATIONS: FROM PROPAGATION MODELS TO SYSTEM PERFORMANCE EVALUATION. IEEE Transactions on Wireless Communications, 2008, 7 (10), 13p. hal-00326363

\section{HAL Id: hal-00326363}

https://hal-centralesupelec.archives-ouvertes.fr/hal-00326363

Submitted on 2 Oct 2008

HAL is a multi-disciplinary open access archive for the deposit and dissemination of scientific research documents, whether they are published or not. The documents may come from teaching and research institutions in France or abroad, or from public or private research centers.
L'archive ouverte pluridisciplinaire HAL, est destinée au dépôt et à la diffusion de documents scientifiques de niveau recherche, publiés ou non, émanant des établissements d'enseignement et de recherche français ou étrangers, des laboratoires publics ou privés. 


\title{
Dual-Polarized Wireless Communications: From Propagation Models to System Performance Evaluation
}

\author{
Claude Oestges, Bruno Clerckx, Maxime Guillaud, and Mérouane Debbah
}

\begin{abstract}
In this paper, we address the potential benefits of dual-polarized arrays in multi-antenna wireless systems. After an extensive literature overview of experimental data, we present a new and simple analytical framework to model dual-polarized Rayleigh and Ricean fading channels for arbitrary array sizes. The model relies on a limited number of physical parameters, such as the channel spatial correlations, the channel co-polar and the cross-polar ratios and the antenna cross-polar discrimination. Then, we investigate the multiplexing advantage of dual-polarized transmissions through the evaluation of the ergodic mutual information, for both TITO and MIMO systems. Finally, the performance of two space-time coding schemes (Alamouti $O$ STBC and uncoded Spatial Multiplexing) is evaluated via a detailed analysis of the pairwise error probability.
\end{abstract}

Index Terms-MIMO, polarization, propagation, mutual information.

\section{INTRODUCTION}

I $\mathrm{N}$ RECENT years, increasing attention has been paid to Multiple-Input Multiple-Output (MIMO) broadband wireless communication systems. However, antenna spacings of at least half a wavelength at the subscriber unit and ten wavelengths at the base station are usually required for achieving significant multiplexing and/or diversity gains. Hence, the use of possibly co-located orthogonally-polarized antennas appears as a space- and cost-effective alternative [1]. Indeed, orthogonal polarizations ideally offer a much better separation between channels, through a large decorrelation at both transmit $(\mathrm{Tx})$ and receive $(\mathrm{Rx})$ sides. In this paper, we consider both Two-Input Two-Output (TITO) and MIMO dualpolarized systems, and we use as a reference the corresponding

Manuscript received May, 22, 2007; revised October 18, 2007 and May, 27, 2008; accepted July, 31, 2008. The associate editor coordinating the review of this paper and approving it for publication was Y. Gong. This work was supported in part by the Belgian Fonds de la Recherche Scientifique, France Telecom R\&D, the EU Network of Excellence NEWCOM and the I0 strategic project of ftw. ftw. is supported by the Austrian Government and by the City of Vienna within the competence center program COMET.

C. Oestges is with the Microwave Laboratory, Université catholique de Louvain, Louvain-la-Neuve, Belgium (e-mail: claude.oestges@uclouvain.be).

B. Clerckx is with the Communication and Networking Laboratory, Samsung Advanced Institute of Technology, Nongseo-Dong, Giheung-Gu, Yongin$\mathrm{Si}$, Gyeonggi-Do, Korea (e-mail: bruno.clerckx@ samsung.com).

M. Guillaud was with the Eurecom Institute, Sophia-Antipolis, France. $\mathrm{He}$ is now with $\mathrm{ftw}$. Forschungszentrum Telekommunikation Wien, Vienna, Austria (e-mail: guillaud@ftw.at).

M. Debbah was with the Eurecom Institute, Sophia-Antipolis, France. $\mathrm{He}$ is now with the Ecole Supérieure d'Electricité (Supelec) - AlcatelLucent Chair on Flexible Radio, Gif-sur-Yvette, France (e-mail: merouane.debbah@supelec.fr).

Digital Object Identifier 10.1109/TWC.2008.070540.
TITO or MIMO uni-polarized system. In the TITO case, both the transmit and receive arrays are made of two spatially separated antennas with orthogonal polarizations, in order to combine both spatial and polarization diversity/multiplexing. The reference scenario is then a TITO configuration with similarly separated uni-polarized antennas. MIMO $n_{r} \times n_{t}$ schemes (for even values of $n_{t}$ and $n_{r}$ ) are modeled by considering that the transmit (resp. receive) array is made of $n_{t} / 2$ (resp. $n_{r} / 2$ ) dual-polarized sub-arrays, extending over a given length $L_{t}\left(\right.$ resp. $\left.L_{r}\right)$. The reference scenario is then a $n_{r} \times n_{t}$ unipolarized scheme based on antenna arrays extending over the same lengths. As can be noticed, we carry out comparisons by keeping constant the dimensions of the MIMO matrix. Indeed, these dimensions equal the number of required RF chains, which are the most expensive components in a system. Note that using two co-located antennas with orthogonal polarizations at both ends of the link already constitute a $2 \times 2$ system. Another definition, motivated by the spatial extension of the antenna arrays, consists in considering the above scenario as a Single-Input Single-Output (SISO) system with dual-polarized antennas. This last definition, which has often been used in the literature, however leads to unfairly comparing a uni-polarized $2 \times 2$ system with a so-called $2 \times 2 \times$ two-polarization system, although the latter is actually a $4 \times 4$ scheme, which needs to be compared with a uni-polarized $4 \times 4$ system if we want to keep the number of RF chains equal.

Despite a number of recent studies focusing on spatial channel models, only a limited number of papers have addressed the polarization issue [1]-[9], theoretically or experimentally, mainly because the (de-)coupling effect between orthogonal polarizations is a complex mechanism. On the one hand, a number of geometry-based models are available for simulating dual-polarized transmissions, the most recent model being used by the 3GPP group [6]. Although these models are very useful in simulating signal processing techniques, they do not allow for analytical manipulations (e.g. in expresions of the mutual information or the error probability). One the other hand, analytical formalisms are needed to design space-time coding and precoding schemes. One can make a comparison with single-polarized schemes: whereas geometrybased models (e.g. the one-ring model of [10]) have been heavily used in simulations, analytical approaches such as the Kronecker [11] or eigenbeam [12] models have proved very useful in code design. Hence, there is an obvious appeal to develop an analytical formalism to model dual-polarized 
multi-antenna channels. The first goal of this paper is precisely to provide a sufficiently general analytical framework using a reduced number of physically sound parameters. Based on this model, it is then possible to clearly identify the benefits of multiple polarizations from a performance point of view. This constitutes the second objective of this paper.

In Section II, we carry out a thorough literature overview of experimental or electromagnetic results regarding dualpolarized channels. These results are then used in Section III to build an analytical model combining the effects of space and polarization separations. In Section IV, we investigate whether dual-polarized systems may possibly increase the system throughput. Finally, in Section V, we analyze the performance of Orthogonal Space-Time Block Coding (OSTBC) and Spatial Multiplexing from an error probability viewpoint.

In this paper, $\mathcal{E}\{x\}=\bar{x}$ represents the expectation of random variable $x,{ }^{*}$ stands for element-wise conjugation, ${ }^{H}$, for conjugate transpose, $x \rightarrow a$ indicates that $x$ tends toward $a, \operatorname{vec}(\mathbf{A})$ is the operator that forms a vector from successive columns of matrix $\mathbf{A},|x|$ is the absolute value of $x,\|A\|_{F}$ is the Frobenius norm of $\mathbf{A}, \operatorname{det}\{\mathbf{A}\}$ is the determinant of matrix $\mathbf{A}, \operatorname{Tr}\{\mathbf{A}\}$ is the trace of matrix $\mathbf{A}, \odot$ is the Hadamard product, $\otimes$ is the Kronecker product, $\mathbf{I}_{n}$ stands for the $n \times n$ identity matrix, and $\mathbf{1}_{n \times m}$ denotes a $n \times m$ matrix with unit entries.

\section{Channel Characterization of Dual-Polarized SYSTEMS}

\section{A. Mechanisms and Parameters}

Ideally, the cross-polar transmissions (e.g. from a verticallypolarized Tx antenna to a horizontally-polarized $\mathrm{Rx}$ antenna) should be equal to zero. This is actually not the case owing to two depolarization mechanisms: the use of imperfect antenna cross-polar isolation (XPI) and the existence of a cross-polar ratio (XPR) in the propagation channel. Both effects combine to yield a global cross-polar discrimination (XPD).

The first mechanism is well-known in antenna theory and is easily accounted for by means of the cross-polar antenna pattern. Analytically, this can be approximated by a coupling matrix at transmit or receive sides

$$
\mathbf{M}_{t}=\left[\begin{array}{cc}
1 & \sqrt{\chi_{a, t}} \\
\sqrt{\chi_{a, t}} & 1
\end{array}\right] \text { and } \mathbf{M}_{r}=\left[\begin{array}{cc}
1 & \sqrt{\chi_{a, r}} \\
\sqrt{\chi_{a, r}} & 1
\end{array}\right]
$$

where the scalar antenna XPI is defined as $\chi_{a, t}^{-1}$ and $\chi_{a, r}^{-1}$ at $\mathrm{Tx}$ and $\mathrm{Rx}$ respectively. Note that antenna XPI is the only mechanism affecting line-of-sight (LOS) components. Scattered components (either coherent or non coherent) are affected by both XPI and XPR, although these are well separated effects if the antenna cross-coupling is represented by $\mathbf{M}_{t}$ and $\mathbf{M}_{r}$.

Throughout this paper, channel matrices are denoted differently, depending on the scenario and the antenna quality, as detailed below:

1) $\mathbf{H}_{\times}$designates the dual-polarized channel matrix combining spatial separation, dual-polarized arrays using antennas with infinite XPI;
2) $\mathbf{H}_{\times, a}=\mathbf{M}_{r} \mathbf{H}_{\times} \mathbf{M}_{t}$ designates the dual-polarized channel matrix combining spatial separation, dual-polarized arrays and finite antenna cross-polar isolation (the subscript $a$ means that antenna XPIs are accounted for);

3) $\mathbf{H}$ designates the uni-polarized channel matrix (all antennas are identically polarized) accounting only for spatial correlations at both ends,

4) $\mathbf{G}$ is the $2 \times 2$ matrix representing the dual-polarized TITO channel for orthogonally-polarized co-located antennas (there are no spatial correlation effects) with infinite XPI, whereas $\mathbf{G}_{a}=\mathbf{M}_{r} \mathbf{G M}_{t}$ is the equivalent matrix, but for antennas with finite XPI.

Furthermore, for Ricean fading channels, the above matrices can be decomposed into a coherent part (we then write $\overline{\mathbf{H}}_{\times}$, $\overline{\mathbf{H}}, \overline{\mathbf{G}}$, etc. in agreement with our notation) and a non-coherent Rayleigh-fading part, proportional to $\tilde{\mathbf{H}}_{\times}, \tilde{\mathbf{H}}, \tilde{\mathbf{G}}$, etc.

Quite arbitrarily, we decided to model the downlink channel (from the base station to the user terminal). Naturally, the uplink channel matrix (from the user terminal to the base station) is simply obtained by transposition of the downlink channel matrix. For dual-polarized arrays with co-located vertically and horizontally-polarized (abbreviated as $\mathrm{VH}$ ) antennas, we write the downlink channel matrix as

$$
\mathbf{G}=\left[\begin{array}{ll}
g_{v v} & g_{v h} \\
g_{h v} & g_{h h}
\end{array}\right] \text {. }
$$

Denoting by $p_{i j}=\left|g_{i j}\right|^{2}$ the instantaneous gain on channel $i j$, we may define various cross-polar ratios [1] (XPDs could be similarly defined based on $\mathbf{G}_{a}$ rather than $\mathbf{G}$ ):

1) uplink cross-polar ratios (uplink XPR)

$$
\begin{aligned}
\mathrm{XPR}_{U^{v}} & =p_{v v} / p_{v h} \\
\mathrm{XPR}_{U^{h}} & =p_{h h} / p_{h v}
\end{aligned}
$$

2) downlink cross-polar ratios (downlink XPR),

$$
\begin{aligned}
\mathrm{XPR}_{D^{v}} & =p_{v v} / p_{h v} \\
\mathrm{XPR}_{D^{h}} & =p_{h h} / p_{v h} .
\end{aligned}
$$

We may also define a unique co-polar ratio as

$$
\mathrm{CPR}=p_{v v} / p_{h h} .
$$

Clearly, all these parameters are not independent. In particular, the following relationships hold true:

$$
\mathrm{CPR}=\frac{\mathrm{XPR}_{U^{v}}}{\mathrm{XPR}_{D^{h}}}=\frac{\mathrm{XPR}_{D^{v}}}{\mathrm{XPR}_{U^{h}}} .
$$

The above definitions only concern channel gains. To define the phase relationships, $\mathbf{G}$ can be characterized by its correlation matrix, given by $\mathcal{E}\left\{\operatorname{vec}\left(\mathbf{G}^{H}\right) \operatorname{vec}\left(\mathbf{G}^{H}\right)^{H}\right\}$. The diagonal elements of this $4 \times 4$ matrix are the various average gains $E\left\{p_{i j}\right\}$, while the off-diagonal elements express the different co-, cross- and anti-polar correlations. The crosspolar correlations (XPC) correspond to the classical transmit and receive correlations, the correlation between $g_{v v}$ and $g_{h h}$ is defined here as the co-polar correlation (CPC), and the correlation between $g_{v h}$ and $g_{h v}$, as the anti-polar correlation (APC). 


\section{B. Overview of Experimental Results}

Most experimental results provide a partial characterization of $\mathbf{G}_{a}$, or even of $\mathbf{H}_{\times, a}$, providing values of the global XPD on the up- or downlink, and rarely of the CPR. ${ }^{1}$ Hence, it is sometimes difficult to isolate the various contributions (antenna XPI, channel XPR and spatial correlations). However, in most cases, one may expect that experimental arrays are made of co-located antennas with a large XPI, and that only significant results (i.e. with XPD lower than the XPI) are reported in these papers. Hence, this implies that for these values, $\mathrm{XPD} \approx \mathrm{XPR}$, and it should be possible to characterize $\mathbf{G}$, at least partially, from the results summarized below. This is the reason why we use XPR denominations in the following, but it should be reminded that these are formally XPD values.

1) Outdoor Scenarios: As far as uplink single-input multiple-output (SIMO) results are concerned, these usually consider a vertically polarized transmitter and a base station using an orthogonally-polarized array with vertical and horizontal polarizations ( $\mathrm{VH}$ ) or 45-degree tilted polarization (slant scheme). For a VH reception, the measured parameters are therefore $\mathrm{XPR}_{U} v$ and the receive cross-polar correlation:

- the experimental uplink XPR in [13] reaches values of $6 \mathrm{~dB}$ and $7.4 \mathrm{~dB}$ respectively in macro- and microcells (at $900 \mathrm{MHz}$ ), with mean envelope cross-polar (receive) correlation coefficients around 0.1 ;

- in [14], the uplink XPR is about $12 \mathrm{~dB}$ in suburban areas and $7 \mathrm{~dB}$ in urban areas at $463 \mathrm{MHz}$, while the power receive correlation is quasi zero;

- in [15], at $1800 \mathrm{MHz}$, the power receive correlations are below 0.3 and the uplink XPR values are around 7 to 9 $\mathrm{dB}$ in various urban and suburban non-LOS scenarios.

In [16], experimental data recorded at $1800 \mathrm{MHz}$ in urban and suburban microcells with handheld terminals highlight that

- the uplink XPR levels approximately equal $7 \mathrm{~dB}$ in urban areas and vary from 8 to $16 \mathrm{~dB}$ in suburban areas for vertical Tx polarization, whereas $\mathrm{XPR}_{U^{h}}$ varies from -4 to $-1 \mathrm{~dB}$ in urban areas and from 2 to $-6 \mathrm{~dB}$ in suburban areas,

- the estimated corresponding CPR levels vary between 5.5 and $4 \mathrm{~dB}$ in urban areas and from 3 to $11 \mathrm{~dB}$ in suburban areas,

- the estimated downlink XPR values are independent from the Tx polarization, and equal approximately $1.5 \mathrm{~dB}$ and $5 \mathrm{~dB}$ respectively in urban and suburban areas.

For \pm 45 degree reception scheme, it is found [14] that the XPR is reduced to $0 \mathrm{~dB}$, but at the expense of a larger receive power correlation $(\approx 0.44)$.

Other experiments have dealt with handheld terminals [17] and have yielded XPR values as low as $2 \mathrm{~dB}$, with mean correlation levels below 0.1 for a $\mathrm{VH}$ reception scheme, and around 0.15 for a slant reception scheme. The low measured $\mathrm{XPR}$ is explained in the paper by interaction between the antenna and the human head.

Let us now consider dual-polarized (MIMO) results. In [1], non-LOS picocell scenarios have been investigated at 1900

\footnotetext{
${ }^{1}$ Many denominations also exist: some papers define XPD/XPR values as horizontal-to-vertical ratios, etc. In all cases, we translated the denominations used in these papers into our own denominations.
}

MHz. However, the use of directional antenna patterns has resulted in different results depending on the array orientation (the orthogonal arrays were placed along a vertical or a horizontal baseline). For the former case, we have that

$$
\mathrm{XPR}_{U^{v}} \approx \mathrm{XPR}_{D^{h}}>>\mathrm{XPR}_{D^{v}} \approx \mathrm{XPR}_{U^{h}},
$$

implying that the CPR is close to $0 \mathrm{~dB}$ via (7), and that, on the average, $p_{v h}<<p_{h v}$. For the horizontal baseline, the situation is as follows:

$$
\mathrm{XPR}_{U^{v}} \approx \mathrm{XPR}_{D^{v}}>>\mathrm{XPR}_{U^{h}} \approx \mathrm{XPR}_{D^{h}},
$$

implying a large CPR and $p_{v h} \approx p_{h v}$ on the average.

In [18], downlink measurement results are presented for a VH-to-VH array scheme at $5.3 \mathrm{GHz}$ in various non-line-ofsight urban environments (corresponding to Rayleigh fading situations). Values of $\mathrm{XPR}_{U^{v}}$ range from 7.6 (macrocells) to $8 \mathrm{~dB}$ (microcells) while the values of $\mathrm{XPR}_{U^{h}}$ range from 2.3 (macrocells) to $6.9 \mathrm{~dB}$ (microcells). Meanwhile, corresponding CPR values range from 1.6 (macrocells) to $0.4 \mathrm{~dB}$ (microcells). Surprisingly, that translates approximately into downlink XPR values quasi-independent of the Tx polarization, especially in microcells (the agreement in microcells is almost perfect). Estimated values of $\mathrm{XPR}_{D^{v}}=\mathrm{XPR}_{D^{h}}$ range from 4 to $6 \mathrm{~dB}$ in macrocells and equal $7.5 \mathrm{~dB}$ in microcells. Transmit and receive cross-polar correlations are almost all equal to 0.3 , but no indication is given about the co- and antipolar correlations.

Results in [19] concern rural areas at 2.5 GHz. The XPR averaged over $\mathrm{XPR}_{D^{v}}$ and $\mathrm{XPR}_{D^{h}}$ (in $\mathrm{dB}$ scale) varies from 2 to $19 \mathrm{~dB}$, with a median of $8.5 \mathrm{~dB}$.

In [9], macrocell propagation data at $1.9 \mathrm{GHz}$ suggest that the ratio between the mean values of $p_{v v}$ and $p_{h h}$ (averaged over the small scale fading) is close to $0 \mathrm{~dB}$, with a standard deviation of $3 \mathrm{~dB}$. Note that this ratio is different from the average ratio between the instantaneous values of $p_{v v}$ and $p_{h h}$. As an example, it is observed that the instantaneous CPR can be as significant as $\pm 15 \mathrm{~dB}$, due to spatial fading decorrelation. The downlink XPR varies from 5 to $15 \mathrm{~dB}$, with an average value of $9 \mathrm{~dB}$, and decreases with the pathloss, reflecting higher XPR in LOS than in NLOS scenarios. The co-, anti-, and cross-polar correlations are very low, but one must mention that co- and anti-polar correlations include a spatial decorrelation effect, as antennas are not all co- located.

2) Indoor Scenarios: In [1], in non-LOS picocells at 1800 $\mathrm{MHz}$, downlink XPR values are about $6.5 \mathrm{~dB}$, irrespective of the Rx polarization, and CPR levels range from -4 to 4 $\mathrm{dB}$. That implies that uplink XPR levels clearly depend on the Tx polarization. In [20], XPR values as low as $3 \mathrm{~dB}$ are measured in an office environment at $2.4 \mathrm{GHz}$. In [21], crosspolar correlations are found to be very small in a typical indoor environment.

In [22], uplink XPR values at $5.2 \mathrm{GHz}$ vary between 7 and $15.7 \mathrm{~dB}$ (for $\mathrm{XPR}_{U^{v}}$ ) and 8.6 to 14.4 (for $\mathrm{XPR}_{U^{h}}$ ). Furthermore, the difference $\mathrm{XPR}_{U^{v}}-\mathrm{XPR}_{U^{h}}$ is not necessarily positive, but may vary from -3 to $+3 \mathrm{~dB}$.

In [7], [8], measurements have been carried out at 5.1 $\mathrm{GHz}$ using a VH scheme at both link ends. The CPR varies from 2 to $6 \mathrm{~dB}$, and the vertical XPR levels are higher than the horizontal XPR values, although the difference 
$\left[\mathrm{XPR}_{U^{v}}-\mathrm{XPR}_{U^{h}}\right]$, which can be as high as $8 \mathrm{~dB}$, is sensibly higher than $\left[\mathrm{XPR}_{D^{v}}-\mathrm{XPR}_{D^{h}}\right]$, which varies between 1 and $4 \mathrm{~dB}$. All correlation coefficients are on average below 0.6, but show a large standard deviation. Finally, indoor results of [9] are very similar to their outdoor results reported above, but with average downlink XPR around $7 \mathrm{~dB}$.

3) Summary: Table I, which summarizes the most complete results, reveals that there is a clear lack of data for dualpolarized channels. Hence, it is somehow not straightforward to infer general conclusions from the above analysis. Yet, some results ( [7], [8], [15], [16], [18], [20], [21] and [1]-indoor) share, at least approximately, the following properties:

- the average CPR usually varies between 0 and $6 \mathrm{~dB}$ in outdoor environments (with maximum values of $11 \mathrm{~dB}$ in suburban areas), and between -4 and $6 \mathrm{~dB}$ indoors; this non-zero value can be easily explained by the polarization selectivity of the reflection and diffraction processes [4], [20],

- there is a clear difference between the vertical and horizontal uplink XPR levels, which are given according to (7) once the downlink XPR and CPR are known,

- from the data, the downlink XPR levels $\mathrm{XPR}_{D^{v}}$ and $\mathrm{XPR}_{D^{h}}$ are often equal (or at least close), with measured values of 4 to $8 \mathrm{~dB}$ in NLOS outdoor cases, up to 15 to $19 \mathrm{~dB}$ in LOS urban and rural areas; 3 to $8 \mathrm{~dB}$ in NLOS indoor cases, and up to $15 \mathrm{~dB}$ in LOS indoor scenarios (note that when the downlink XPR values have not been measured, we have estimated them based on (7)),

- the cross-polar correlations (XPC) are usually small,

- the co- and anti-polar correlations (CPC/APC) seem to be small (but the results are really scarce).

\section{Output from Theoretical Models}

Electromagnetic models yield some precious insight into dual-polarized propagation. As an example, a ray-tracing tool [23] has been applied in a macrocellular urban environment at $2.4 \mathrm{GHz}$, with the receiver moving along a street perpendicular to the link axis. The ray-tracing tool accounts for reflections up to the third order, as well as wedge and corner diffraction. It is found that the narrowband channel for a $2 \times 2$ dual-polarized $\mathrm{VH}-\mathrm{to}-\mathrm{VH}$ transmission has the following properties:

- the CPR is lognormally distributed with an average value of 4 to $7 \mathrm{~dB}$,

- the downlink XPRs are identical for both $v$ and $h$ polarizations, being lognormally distributed with an average value of $12 \mathrm{~dB}$,

- the transmit and receive cross-polar correlations are small,

- the co-polar and anti-polar correlations vary between 0.8 and 0.9 .

Naturally, ray-tracing cannot account for smaller and nonspecular obstacles, which are expected to provide lower CPR and XPR levels. Other papers have reached similar conclusions, using rough scattering models [24]. Note that the first three properties are in line with the experimental campaigns, while the last one is not (but there are only a few available data to compare with).

\section{Channel Models For DuAl-Polarized SystemS}

\section{A. Dual-Polarized TITO Rayleigh Fading Channels}

In Rayleigh fading, it has been observed [25, p. 109] that the spatial correlation properties (modeled by $\tilde{\mathbf{H}}$ and $\mathbf{R}$ ) are independent of the polarization, especially in macro- and microcells, provided that the direction spectra are similar for all antennas. This namely implies that, if antennas are directional, they should be similarly oriented. In other words, the CPR (resp. XPR) measured on $\tilde{\mathbf{H}}_{\times}$should be equal to the CPR (resp. XPR) measured on $\tilde{\mathbf{G}}$. If this assumption holds true, the dual-polarized Rayleigh channel matrix with spatially-separated antennas may be rewritten as

$$
\tilde{\mathbf{H}}_{\times}=\tilde{\mathbf{H}} \odot \tilde{\mathbf{X}} .
$$

In (10), $\tilde{\mathbf{H}}$ is modeled as a uni-polarized correlated Rayleigh channel, while $\tilde{\mathbf{X}}$ models both the correlation and power imbalance impacts of the channel depolarization. It is important to note that it would be incorrect to write $\tilde{\mathbf{H}}_{\times}=\tilde{\mathbf{H}} \odot \tilde{\mathbf{G}}$. Indeed, this expression contains twice a fading term, in both $\tilde{\mathbf{H}}$ and $\tilde{\mathbf{G}}$. Hence, we have introduced a new matrix $\tilde{\mathbf{X}}$, whose goal is to model only the power imbalance and the phase-shifts between the four channels. The relationship between $\tilde{\mathbf{X}}$ and $\tilde{\mathbf{G}}$ is however very simple, as $\tilde{\mathbf{G}}=g \tilde{\mathbf{X}}$, where $g$ is a scalar complex Gaussian term representing fading.

In (10), multipath fading is entirely modeled by $\tilde{\mathbf{H}}$, using the classical expression [26]

$$
\operatorname{vec}\left(\tilde{\mathbf{H}}^{H}\right)=\mathbf{R}^{1 / 2} \operatorname{vec}\left(\tilde{\mathbf{H}}_{w}^{H}\right),
$$

where $\tilde{\mathbf{H}}_{w}$ is the classical i.i.d. complex Gaussian matrix and $\mathbf{R}=\mathcal{E}\left\{\operatorname{vec}\left(\tilde{\mathbf{H}}^{H}\right) \operatorname{vec}\left(\tilde{\mathbf{H}}^{H}\right)^{H}\right\}$ is a $4 \times 4$ spatial correlation matrix,

$$
\mathbf{R}=\left[\begin{array}{cccc}
1 & t^{*} & r^{*} & s_{1}^{*} \\
t & 1 & s_{2}^{*} & r^{*} \\
r & s_{2} & 1 & t^{*} \\
s_{1} & r & t & 1
\end{array}\right]
$$

with $t$ and $r$ being the transmit and receive antenna correlations, and $s_{1}=\mathcal{E}\left\{\tilde{\mathbf{H}}(1,1) \tilde{\mathbf{H}}^{*}(2,2)\right\}$ and $s_{2}=$ $\mathcal{E}\left\{\tilde{\mathbf{H}}(1,2) \tilde{\mathbf{H}}^{*}(2,1)\right\}$ being the cross-channel correlations [27].

To model $\tilde{\mathbf{X}}$ for VH-to-VH downlink transmissions, we resort to the results of Section II. Hence, a fairly general model accounting for the generally observed trends summarized above and satisfying (7) is given by

$$
\begin{aligned}
& \operatorname{vec}\left(\tilde{\mathbf{X}}^{H}\right)= \\
& {\left[\begin{array}{cccc}
1 & \sqrt{\mu \chi} \vartheta^{*} & \sqrt{\chi} \sigma^{*} & \sqrt{\mu} \delta_{1}^{*} \\
\sqrt{\mu \chi} \vartheta & \mu \chi & \sqrt{\mu} \chi \delta_{2}^{*} & \mu \sqrt{\chi} \sigma^{*} \\
\sqrt{\chi} \sigma & \sqrt{\mu} \chi \delta_{2} & \chi & \sqrt{\mu \chi} \vartheta^{*} \\
\sqrt{\mu} \delta_{1} & \mu \sqrt{\chi} \sigma & \sqrt{\mu \chi} \vartheta & \mu
\end{array}\right] \operatorname{vec}\left(\tilde{\mathbf{X}}_{w}^{H}\right),}
\end{aligned}
$$

where

- $\mu$ and $\chi$ represent the inverse of, respectively, the co-polar and the downlink cross-polar ratios (CPR and $\mathrm{XPR}_{D^{v}}=$ $\mathrm{XPR}_{D^{h}}$ ) and are assumed to be constant in this paper,

- $\sigma$ and $\vartheta$ are the receive and transmit correlation coefficients for co-located antennas (i.e. the correlation 
TABLE I

OVERVIEW OF EXPERIMENTAL RESULTS (XPR AND CPR VALUES ARE EXPRESSED IN DECIBELS)

\begin{tabular}{c|c|c|c|c|c|c|c} 
Scenario & Freq. [GHz] & $\mathrm{XPR}_{U^{v}}$ & $\mathrm{XPR}_{U^{h}}$ & $\mathrm{CPR}$ & $\mathrm{XPR}_{D}$ & $\mathrm{XPC}$ & $\mathrm{CPC} / \mathrm{APC}$ \\
\hline Suburban [14] & 0.46 & 12 & - & - & - & $\approx 0$ & - \\
Urban [14] & 0.46 & 7 & - & - & - & $\approx 0$ & - \\
Macrocell [13] & 0.9 & 6 & - & - & - & 0.1 & - \\
Microcell [13] & 0.9 & 7.4 & - & - & - & 0.1 & - \\
Suburban/urban NLOS [15] & 1.8 & 7 to 9 & - & - & - & $\leq 0.3$ & - \\
Urban microcell [16] & 1.8 & 7 & -4 to -1 & 4 to 5.5 & 1.5 & - & - \\
Suburban microcell [16] & 1.8 & 8 to 16 & -6 to -2 & 3 to 11 & 5 & - & - \\
Urban NLOS microcell [18] & 5.3 & 8 & 2.3 & 1.6 & 7.5 & $\approx 0.3$ & - \\
Urban NLOS macrocell [18] & 5.3 & 7.6 & 6.9 & 0.4 & 4 to 6 & $\approx 0.3$ & - \\
Urban macrocell [9] & 1.9 & 9 & 9 & 0 & 9 & $\approx 0$ & $\approx 0$ \\
Indoor [9] & 1.9 & 7 & 7 & 0 & 7 & $\approx 0$ & $\approx 0$ \\
Indoor NLOS picocell [1] & 1.8 & 2.5 to 10.5 & - & -4 to 4 & 6.5 & - & - \\
Indoor picocell [22] & 5.2 & 7 to 15.7 & 8.6 to 14.4 & - & - & - & - \\
Indoor corridor/hall [7], [8] & 5.1 & 7 to 9.5 & 1.5 to 6.7 & 2.5 to 6 & 8 to $11(v)$ & $\leq 0.6$ & $\leq 0.6$
\end{tabular}

coefficients between $v v$ and $h v, h h$ and $h v, v v$ and $v h$ or $h h$ and $v h$ ),

- $\delta_{1}$ and $\delta_{2}$ are the co- and anti-polar correlation coefficients,

- $\tilde{\mathbf{X}}_{w}$ is a $2 \times 2$ matrix whose four elements are independent circularly symmetric complex exponentials of unit amplitude, $e^{j \phi_{k}}, k=1, \ldots, 4$, the angles $\phi_{k}$ being uniformly distributed over $[0,2 \pi)$.

Note that this model does not make any simplification on the polarization-induced correlations, but only assumes that the downlink XPD levels are independent from the transmit polarization. Values of $\mu$ and $\chi$ naturally depend on the considered environment, as outlined in Section II.

In the performance analysis, we consider (only for simplicity) that the cross-polar correlation coefficients $\sigma$ and $\vartheta$ are equal to zero, as often observed experimentally. As far as $\delta_{1}$ and $\delta_{2}$ are concerned, our overview does not allow us to assign definite values. Hence, we will consider only two cases in this paper, which seem both reasonable assumptions from the literature overview: $\left|\delta_{1}\right|=1,\left|\delta_{2}\right|=1$, as found by the ray-tracing simulations [23], or $\delta_{1}=\delta_{2}=0$, as suggested by a few results as well as some recent studies [28] (note that the system performance is usually not very sensitive to correlation for correlations lower than 0.6).

Since $\tilde{\mathbf{X}}$ is a random matrix, the two eigenvalues of $\tilde{\mathbf{X}} \tilde{\mathbf{X}}^{H}$, denoted as $\eta_{1}$ and $\eta_{2}$ are also random, and can be explicitly written as a function of the deterministic parameters $\mu, \chi$, $\delta_{1}$ and $\delta_{2}$ on the one hand, and of the random phase shifts $\phi_{1}$ to $\phi_{4}$ on the other hand. As an example, if $\mu=1$, these eigenvalues read as

$$
\eta_{1,2}=A \pm \sqrt{A^{2}+B},
$$

where

$$
\begin{aligned}
A & =1+\chi+\chi\left|\delta_{2}\right| \sqrt{1-\left|\delta_{2}\right|^{2}} \cos \left(\phi_{2}-\phi_{3}+\arg \left\{\delta_{2}\right\}\right) \\
& +\left|\delta_{1}\right| \sqrt{1-\left|\delta_{1}\right|^{2}} \cos \left(\phi_{1}-\phi_{4}+\arg \left\{\delta_{1}\right\}\right)
\end{aligned}
$$

and

$$
\begin{aligned}
B & =2 \chi\left|\delta_{2}\right|\left|\delta_{1}\right| \cos \left(2 \phi_{1}-2 \phi_{2}+\arg \left\{\delta_{1}\right\}-\arg \left\{\delta_{2}\right\}\right) \\
& +\chi^{2}\left|\delta_{1}\right| \sqrt{1-\left|\delta_{2}\right|^{2}} \cos \left(2 \phi_{1}-\phi_{2}-\phi_{3}+\arg \left\{\delta_{1}\right\}\right) \\
& +2 \chi\left|\delta_{2}\right| \sqrt{1-\left|\delta_{1}\right|^{2}} \cos \left(\phi_{1}-2 \phi_{2}+\phi_{4}-\arg \left\{\delta_{2}\right\}\right) \\
& +2 \chi^{2} \sqrt{1-\left|\delta_{2}\right|^{2}} \sqrt{1-\left|\delta_{1}\right|^{2}} \cos \left(\phi_{1}-\phi_{2}-\phi_{3}+\phi_{4}\right) \\
& -2\left|\delta_{1}\right| \sqrt{1-\left|\delta_{1}\right|^{2}} \cos \left(\phi_{1}-\phi_{4}+\arg \left\{\delta_{1}\right\}\right)-1-\chi^{2} \\
& -2 \chi^{2}\left|\delta_{2}\right| \sqrt{1-\left|\delta_{2}\right|} \cos \left(\phi_{2}-\phi_{3}+\arg \left\{\delta_{2}\right\}\right) .
\end{aligned}
$$

Note that if $\delta_{1}=\delta_{2}=0$, the eigenvalues further simplify into

$$
\eta_{1,2}=1+\chi \pm \sqrt{2 \chi(1+\cos \psi)},
$$

where $\psi=\phi_{1}-\phi_{2}-\phi_{3}+\phi_{4}$ is defined as a random angle uniformly distributed over $[0,2 \pi)$.

Interestingly, the model of (10) differs from the model used in [29], where $\tilde{\mathbf{H}}_{\times}$is decomposed by extracting the impact of depolarization on the channel gains, yielding

$$
\tilde{\mathbf{H}}_{\times}=\hat{\mathbf{H}} \odot|\tilde{\mathbf{X}}|,
$$

where $|\tilde{\mathbf{X}}|$ depends on the polarization scheme. What is important to notice is that $\hat{\mathbf{H}}$ still includes two correlation mechanisms (space and polarization). Hence, it is generally not equal to an equivalent uni-polarized transmission matrix $\tilde{\mathbf{H}}$ (i.e. with the same antenna spacings, all polarizations being then identical). As a result, $\hat{\mathbf{H}}$ is some hybrid matrix, modeling the correlation aspects of both spacing and polarization. In that sense, it is impossible to use this model to compare uniand dual-polarized schemes. By contrast, our model operates the decomposition into two matrices based on the physical mechanisms (space versus polarization) rather than on their impact (gain versus correlation).

\section{B. Dual-Polarized TITO Ricean Fading Channels}

Defining the Ricean K-factor on the $v v$ reference transmission as $K$, and assuming the LOS is the only coherent contribution (typically, in mobile scenarios), the global Ricean fading channel matrix, also accounting for antenna XPIs, is 
given by

$$
\mathbf{H}_{\times, a}=\underbrace{\mathbf{M}_{r} \overline{\mathbf{H}}_{\times} \mathbf{M}_{t}}_{\overline{\mathbf{H}}_{\times, a}}+\sqrt{\frac{1}{1+K}} \underbrace{\mathbf{M}_{r} \tilde{\mathbf{H}}_{\times} \mathbf{M}_{t}}_{\tilde{\mathbf{H}}_{\times, a}}
$$

where $\overline{\mathbf{H}}_{\times}$reads, for a system having the same $\mathrm{VH}$ polarization scheme at both $\mathrm{Tx}$ and $\mathrm{Rx}$, as

$$
\overline{\mathbf{H}}_{\times} \triangleq \overline{\mathbf{X}}=\sqrt{\frac{K}{1+K}}\left[\begin{array}{cc}
e^{j \varsigma_{v v}} & 0 \\
0 & e^{j \varsigma_{h h}}
\end{array}\right],
$$

with $\varsigma_{v v}$ and $\varsigma_{h h}$ related to the baseline array orientation (e.g. $\varsigma_{v v}=\varsigma_{h} h=0$ for broadside arrays at both ends, so $\overline{\mathbf{X}}=\sqrt{\frac{K}{1+K}} \mathbf{I}_{2}$ in such cases). Note that (19) does not result in equal $\mathrm{K}$-factors for all channels, as the powers of the individual Rayleigh channels are not equal via (13).

\section{Dual-Polarized MIMO Fading Channels}

As already mentioned, we analyze particular $n_{r} \times n_{t}$ schemes (for even values of $n_{t}$ and $n_{r}$ ) for which the Tx and $\mathrm{Rx}$ array are made of $n_{t} / 2$ and $n_{r} / 2$ dual-polarized sub-arrays (each sub-array is identical and is made of two co-located antennas with orthogonal polarizations). If we further assume that all sub-arrays are identically oriented, the Rayleigh channel matrix is easily written as

$$
\tilde{\mathbf{H}}_{\times, n_{r} \times n_{t}}=\tilde{\mathbf{H}}_{n_{r} / 2 \times n_{t} / 2} \otimes \tilde{\mathbf{X}}
$$

where the covariance of $\tilde{\mathbf{H}}_{n_{r} / 2 \times n_{t} / 2}$ is the spatial covariance related to the spacing between the sub-arrays, and $\tilde{\mathbf{X}}$ is the $2 \times 2$ dual-polarized matrix modeled by (13). Again, $\tilde{\mathbf{X}}$ only models the differential attenuation and the correlated phaseshifts between the four dual-polarized channels. Note that (21) is valid if and only if the joint transmit-receive direction spectrum is identical for all sub-arrays (they "see" the same scattering environment). This implies e.g. that the channel as seen by PIFA-like designs [30] cannot be modeled by (21).

The Ricean contribution is represented (for a LOS component and broadside linear arrays) by

$$
\overline{\mathbf{H}}_{\times, n_{r} \times n_{t}}=\mathbf{1}_{n_{r} / 2 \times n_{t} / 2} \otimes \overline{\mathbf{X}} .
$$

Eventually, antenna XPIs are included in the above model as follows

$$
\begin{aligned}
\mathbf{H}_{\times, a, n_{r} \times n_{t}} & =\mathbf{1}_{n_{r} / 2 \times n_{t} / 2} \otimes \mathbf{M}_{r} \overline{\mathbf{X}} \mathbf{M}_{t} \\
& +\sqrt{\frac{1}{1+K}} \tilde{\mathbf{H}}{ }_{n_{r} / 2 \times n_{t} / 2} \otimes \mathbf{M}_{r} \tilde{\mathbf{X}} \mathbf{M}_{t}
\end{aligned}
$$

Finally, a few words about power normalization [28]. The above channel models consider that the channel energy on the $v v$ link is one, so that the receive SNR does not depend on the amount of depolarization. If we use a fixed transmit power constraint, we define the so-called transmit SNR on the $v v$ reference link as $\rho_{0}$, so that the receive $\mathrm{SNR}$ is equal to

$$
\rho=\rho_{0} \frac{1}{1+\chi_{a, r}} \frac{K+1}{K+\chi+1} \frac{1}{1+\chi_{a, t}},
$$

which expresses that if the transmit power is fixed, the receive SNR will decrease for smaller XPI and/or XPR. Naturally, this normalization issue only appears when comparing dualpolarized transmissions with different XPI and/or XPR.

\section{Mutual Information OF DUAL-Polarized CHANNELS}

\section{A. TITO Channels}

For very high $\mathrm{K}$-factors, the channel matrix only depends on the Ricean component. Assuming that the Tx antennas have a perfect cross-polar isolation $\left(\chi_{a, t}=0\right)$, we have that

$$
\mathbf{H}_{\times, a} \approx \mathbf{M}_{r} \overline{\mathbf{H}}_{\times}=\left[\begin{array}{cc}
1 & \sqrt{\chi_{a, r}} \\
\sqrt{\chi_{a, r}} & 1
\end{array}\right] .
$$

Let us consider two particular cases, i.e. $\chi_{a, r}=0$ (infinite $\mathrm{XPI}$ ) and $\chi_{a, r}=1$ (no isolation). The mutual information with equal power allocation and fixed transmit power is then given by

$$
\begin{aligned}
\mathcal{I}_{\times}\left(\chi_{a, r}=0\right) & =2 \log _{2}\left(1+\frac{\rho_{0}}{2}\right) \\
\left.\mathcal{I}_{(} \chi_{a, r}=0\right) & =\log _{2}\left(1+2 \rho_{0}\right) \\
\mathcal{I}_{\times}\left(\chi_{a, r}=1\right) & =\mathcal{I}\left(\chi_{a, r}=1\right)=\log _{2}\left(1+\rho_{0}\right),
\end{aligned}
$$

where $\mathcal{I}_{\times}$refers to dual-polarized schemes, and $\mathcal{I}$, to unipolarized schemes. It is clear that, for both uni- and dualpolarized schemes, the mutual information increases with antenna XPI, as a small XPI means a constant channel energy but a lower rank for dual-polarized schemes, a and a constant rank (of one) but a lower channel energy for uni-polarized schemes. This also implies that for large XPI (i.e. $\chi_{a, r} \rightarrow 0$ ), the mutual information is larger for dual-polarized schemes than for uni-polarized schemes when the SNR is larger than a given threshold [26].

We assume now a finite $\mathrm{K}$-factor, and use the model of (13) to highlight the impact of co- and cross-polar ratios on a mutual information upper bound. For convenience, we take $\chi_{a, t}=\chi_{a, r}=0$ and consider two scenarios:

- spatially correlated antennas and $\delta_{1}=1,\left|\delta_{2}\right|=1$,

$$
\begin{gathered}
\overline{\mathbf{H}}_{\times}=\sqrt{\frac{K}{K+1}} \mathbf{I}_{2} \\
\tilde{\mathbf{H}}_{\times}=\left[\begin{array}{cc}
1 & \sqrt{\mu \chi} e^{j\left(\phi+\arg \left\{\delta_{2}\right\}\right)} \\
\sqrt{\chi} e^{j \phi} & \sqrt{\mu}
\end{array}\right] \odot \tilde{\mathbf{H}}
\end{gathered}
$$

where $\tilde{\mathbf{H}}$ is given by (11) and $\phi$ is a random angle uniformly distributed over $[0,2 \pi)$,

- well separated (uncorrelated) antennas,

$$
\begin{gathered}
\overline{\mathbf{H}}_{\times}=\sqrt{\frac{K}{K+1}} \mathbf{I}_{2} \\
\tilde{\mathbf{H}}_{\times}=\left[\begin{array}{cc}
1 & \sqrt{\mu \chi} \\
\sqrt{\chi} & \sqrt{\mu}
\end{array}\right] \odot \tilde{\mathbf{H}}_{w}
\end{gathered}
$$

which is valid irrespective of $\delta_{1}$ and $\delta_{2}$.

Two alternative scenarios (i.e. $\delta_{1}=\delta_{2}=0$ with close or well separated antennas) do not need being considered, as they are indeed both covered by the second scenario (all three scenarios cause elements of $\tilde{\mathbf{H}}_{\times}$to become fully uncorrelated). For both analyzed scenarios, we resort to an upper bound [27] of the ergodic mutual information with equal power allocation $\overline{\mathcal{I}}$, outlined by

$$
\overline{\mathcal{I}} \leq \log _{2}(\bar{\kappa})=\log _{2}\left(\mathcal{E}\left\{\operatorname{det}\left[\mathbf{I}_{2}+\frac{\rho}{2} \mathbf{H}_{\times} \mathbf{H}_{\times}^{H}\right]\right\}\right),
$$

where the receive SNR is given by $\rho=\rho_{0}(K+1) /(K+\chi+1)$. 


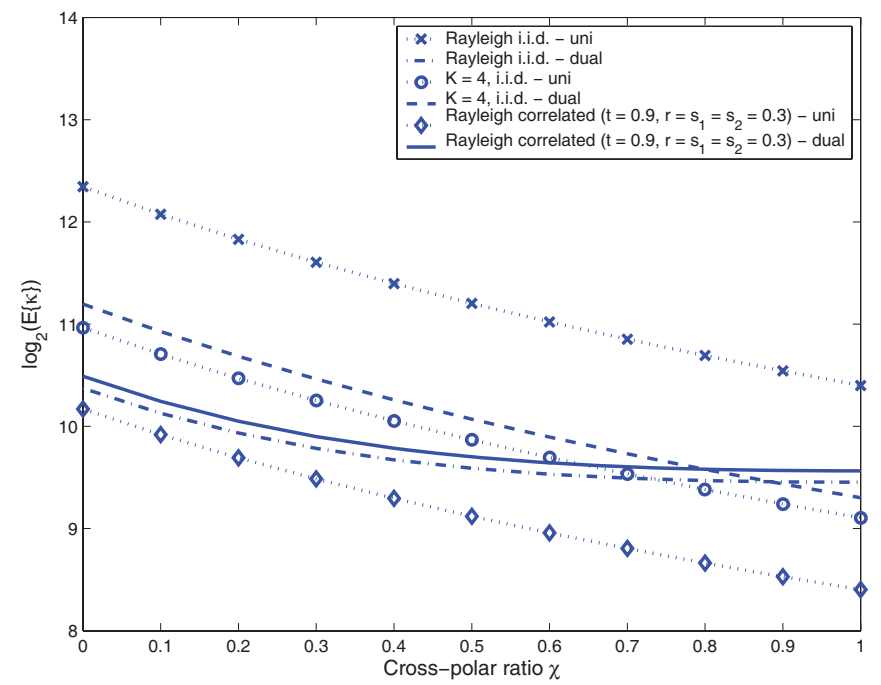

Fig. 1. Mutual information of uni- and dual-polarized $2 \times 2$ channels for different K-factors, correlations and cross-polar ratios

In the considered cases, the upper bounds become

$$
\begin{gathered}
\log _{2}(\bar{\kappa})=\log _{2}\left\{1+\frac{\rho}{2}\left[\frac{2 K+(1+\mu)(1+\chi)}{K+1}\right]\right. \\
+\left(\frac{\rho}{2}\right)^{2} \frac{1}{(K+1)^{2}}\left[K\left(K+2 \Re\left[s_{1} \delta_{1}\right] \sqrt{\mu}+\mu+1\right)\right. \\
\left.\left.+\mu\left(1+\left|s_{1} \delta_{1}\right|^{2}+\chi^{2}\left(1+\left|s_{2} \delta_{2}\right|^{2}\right)\right)\right]\right\} .
\end{gathered}
$$

We first observe that if $K \rightarrow \infty$, (32) approaches the result of (26) in both considered cases. Then we also note that the mutual information is higher in the first scenario (i.e. $\left|\delta_{1}\right|=$ $\left.1,\left|\delta_{2}\right|=1\right)$. Indeed, in the latter, the channel matrix behaves as a diagonal channel (for $\mu=\chi=1$ and $K=0, \mathbf{H}_{\times}=\tilde{\mathbf{H}}_{\times}$ is exactly a $2 \times 2$ diagonal channel [31]). Note however that the difference between achieved mutual informations in both scenarios remains limited. Finally, for a fixed transmit SNR $\rho_{0}$, the ergodic mutual information of Rayleigh channels decreases in (32) as $\mu$ and $\chi$ increase, as illustrated in Figure 1 as far as $\chi$ is concerned (taking $\mu=0.5$ and close antennas with $\left|\delta_{1}\right|=$ $\left.\left|\delta_{2}\right|=1\right)$. This is caused by the fixed transmit power constraint (the opposite result is found when keeping the receive SNR constant). We also observe that dual-polarized transmissions only offer a larger mutual information for Ricean or highly correlated Rayleigh fading channels.

\section{B. MIMO Channels}

When considering an arbitrary array size, the number of parameters of the spatial correlation matrix rapidly increases. To obtain meaningful results, we therefore make the following simplifications:

- we use the same number of antennas on both sides $\left(n_{t}=\right.$ $\left.n_{r}=n\right)$,

- we restrict our analysis to uniform linear arrays of lengths $L_{t}$ and $L_{r}$ (respectively for the transmit and receive arrays), with antenna correlations being exponential functions of the spacings $\left(d_{t}\right.$ and $\left.d_{r}\right)$ [32], i.e. they are proportional to $e^{-d_{t} / \Delta_{t}}$ at the $\mathrm{Tx}$ side, and $e^{-d_{r} / \Delta_{r}}$ at the $\mathrm{Rx}$ side $\left(\Delta_{t}\right.$ and $\Delta_{r}$ are characteristic distances proportional to the spatial coherence distance at each side),

- we take $K=0$,

- we finally assume that the spatial correlation matrix $\mathbf{R}$ is separable (i.e. the well-known Kronecker model may be used to represent $\mathbf{R}$ ).

As a consequence of the above assumptions, the Tx and Rx correlation matrices are respectively expressed as

$$
\begin{aligned}
& \Theta_{t}= \\
& {\left[\begin{array}{cccc}
1 & e^{-d_{t} / \Delta_{t}} & \ldots & e^{-(n-1) d_{t} / \Delta_{t}} \\
e^{-d_{t} / \Delta_{t}} & 1 & \ldots & e^{-(n-2) d_{t} / \Delta_{t}} \\
\vdots & & \ddots & \\
e^{-(n-1) d_{t} / \Delta_{t}} & e^{-(n-2) d_{t} / \Delta_{t}} & \ldots & 1
\end{array}\right]}
\end{aligned}
$$

$$
\begin{aligned}
& \Theta_{r}= \\
& {\left[\begin{array}{cccc}
1 & e^{-d_{r} / \Delta_{r}} & \ldots & e^{-(n-1) d_{r} / \Delta_{r}} \\
e^{-d_{r} / \Delta_{r}} & 1 & \ldots & e^{-(n-2) d_{r} / \Delta_{r}} \\
\vdots & & \ddots & \\
e^{-(n-1) d_{r} / \Delta_{r}} & e^{-(n-2) d_{r} / \Delta_{r}} & \ldots & 1
\end{array}\right],}
\end{aligned}
$$

while the full correlation matrix simply reads as $\mathbf{R}=\Theta_{r} \otimes \Theta_{t}$. It can also be shown that the determinant of, say, $\boldsymbol{\Theta}_{t}$ reads as

$$
\operatorname{det} \Theta_{t}=\left(1-e^{-2 d_{t} / \Delta_{t}}\right)^{n-1}=\left(1-e^{-\frac{2 L_{t}}{(n-1) \Delta_{t}}}\right)^{n-1}
$$

where $d_{t}=L_{t} /(n-1)$ is the element spacing for $n$ antennas over a length $L_{t}$.

Since our goal is to compare uni-polarized with dualpolarized systems, we consider two systems: the reference system is made of uni-polarized arrays with $n$ equi-spaced antennas whereas the second system is made of $n / 2$ dualpolarized equi-spaced sub-arrays. The channel matrices therefore read as

- for the first system,

$$
\tilde{\mathbf{H}}=\boldsymbol{\Theta}_{r}^{1 / 2} \tilde{\mathbf{H}}_{w} \boldsymbol{\Theta}_{t}^{1 / 2},
$$

- for the second system,

$$
\tilde{\mathbf{H}}_{\times}=\underbrace{\boldsymbol{\Theta}_{r}^{\prime 1 / 2} \tilde{\mathbf{H}}_{w}^{\prime} \boldsymbol{\Theta}_{t}^{\prime 1 / 2}}_{\tilde{\mathbf{H}}^{\prime}} \otimes \tilde{\mathbf{X}}
$$

Note that $\boldsymbol{\Theta}_{r}, \tilde{\mathbf{H}}_{w}$ and $\boldsymbol{\Theta}_{t}$ are $n \times n$ matrices, while $\boldsymbol{\Theta}^{\prime}{ }_{r}$, $\tilde{\mathbf{H}}^{\prime}{ }_{w}$ and $\boldsymbol{\Theta}^{\prime}{ }_{t}$ are $n / 2 \times n / 2$. It is also interesting to note that

$$
\tilde{\mathbf{H}}_{\times} \tilde{\mathbf{H}}_{\times}^{H}=\tilde{\mathbf{H}}^{\prime} \tilde{\mathbf{H}}^{\prime H} \otimes \tilde{\mathbf{X}} \tilde{\mathbf{X}}^{H}
$$

Finally, we may arbitrarily use a fixed receive or transmit power constraint, as our goal is to compare uni- and dualpolarized schemes with given $\chi$ and $\mu$.

1) High SNR Analysis: The mutual information of channel $\mathbf{H}$ using an identity transmit covariance is well approximated 
at high SNR [26] by

$$
\mathcal{I} \approx \log _{2} \operatorname{det}\left[\frac{\rho}{n} \mathbf{H} \mathbf{H}^{H}\right] .
$$

For uni-polarized systems, (39) can be developed as follows:

$$
\begin{aligned}
\mathcal{I} & \approx \log _{2}\left\{\left(\frac{\rho}{n}\right)^{n} \operatorname{det}\left[\tilde{\mathbf{H}} \tilde{\mathbf{H}}^{H}\right]\right\} \\
& =n \log _{2}\left(\frac{\rho}{n}\right)+\log _{2} \operatorname{det} \boldsymbol{\Theta}_{r}+\log _{2} \operatorname{det} \Theta_{t} \\
& +\log _{2} \operatorname{det}\left[\tilde{\mathbf{H}}_{w} \tilde{\mathbf{H}}_{w}^{H}\right] \\
& =n \log _{2}\left(\frac{\rho}{n}\right)+(n-1) \log _{2}\left[1-e^{-\frac{2 L_{t}}{(n-1) \Delta_{t}}}\right] \\
& +(n-1) \log _{2}\left[1-e^{-\frac{2 L_{r}}{(n-1) \Delta_{r}}}\right] \\
& +\log _{2} \operatorname{det}\left[\tilde{\mathbf{H}}_{w} \tilde{\mathbf{H}}_{w}^{H}\right] .
\end{aligned}
$$

The ergodic mutual information is then given by

$$
\begin{aligned}
\overline{\mathcal{I}} & =\mathcal{E}\{\mathcal{I}\}=n \log _{2}\left(\frac{\rho}{n}\right) \\
& +(n-1) \log _{2}\left[\left(1-e^{-\frac{2 L_{t}}{(n-1) \Delta_{t}}}\right)\left(1-e^{-\frac{2 L_{r}}{\left(n-1 \Delta_{r}\right.}}\right)\right] \\
& +\frac{1}{\log 2}\left(\sum_{k=1}^{n} \sum_{l=1}^{n-k} \frac{1}{l}-n \gamma\right),
\end{aligned}
$$

where $\gamma \approx 0.57721566$ is Euler's constant. For dual-polarized systems in the high SNR regime, we have:

$$
\begin{aligned}
\mathcal{I}_{\times} & \approx n \log _{2}\left(\frac{\rho}{n}\right)+\frac{n}{2} \log _{2}\left(\eta_{1} \eta_{2}\right) \\
& +2 \log _{2} \operatorname{det}\left[\tilde{\mathbf{H}}_{w}^{\prime}\left(\tilde{\mathbf{H}}_{w}^{\prime}\right)^{H}\right] \\
& +2\left(\frac{n}{2}-1\right) \log _{2}\left[1-e^{-\frac{4 L_{t}}{(n-2) \Delta_{t}}}\right] \\
& +2\left(\frac{n}{2}-1\right) \log _{2}\left[1-e^{-\frac{4 L_{r}}{(n-2) \Delta_{r}}}\right],
\end{aligned}
$$

so that the ergodic mutual information becomes

$$
\begin{aligned}
\overline{\mathcal{I}}_{\times} & =\mathcal{E}\left\{\mathcal{I}_{\times}\right\}=n \log _{2}\left(\frac{\rho}{n}\right)+\frac{n}{2} \mathcal{E}\left\{\log _{2}\left(\eta_{1} \eta_{2}\right)\right\} \\
& +\frac{2}{\log 2}\left(\sum_{k=1}^{n / 2} \sum_{l=1}^{n / 2-k} \frac{1}{l}-\frac{n}{2} \gamma\right) \\
& +2\left(\frac{n}{2}-1\right) \log _{2}\left[1-e^{-\frac{4 L_{t}}{(n-2) \Delta_{t}}}\right] \\
& +2\left(\frac{n}{2}-1\right) \log _{2}\left[1-e^{-\frac{4 L_{r}}{(n-2) \Delta_{r}}}\right]
\end{aligned}
$$

We are now able to calculate the normalized difference $\Delta \overline{\mathcal{I}} / n=\left(\overline{\mathcal{I}}_{\times}-\overline{\mathcal{I}}\right) / n$ assuming that $n$ is large. To simplify the notations, let us define $\xi_{t}=n \Delta_{t} / L_{t}$ and $\xi_{r}=n \Delta_{r} / L_{r}$, which can be thought of as normalized antenna densities at
Tx and Rx. This yields

$$
\begin{aligned}
\Delta \overline{\mathcal{I}} / n & \approx 1+\log _{2}\left[\frac{1-e^{-4 / \xi_{t}}}{1-e^{-2 / \xi_{t}}}\right]+\log _{2}\left[\frac{1-e^{-4 / \xi_{r}}}{1-e^{-2 / \xi_{r}}}\right] \\
& +\frac{1}{2} \mathcal{E}\left\{\log _{2}\left(\eta_{1} \eta_{2}\right)\right\} .
\end{aligned}
$$

Furthermore, if $\delta_{1}$ and $\delta_{2}$ are sufficiently small (typically, lower than 0.25), numerical evaluations show that $\mathcal{E}\left\{\log _{2}\left(\eta_{1} \eta_{2}\right)\right\} \approx 0$, irrespective of $\chi$ and $\mu$. As an example, if $\delta_{1}=\delta_{2}=0$ and $\mu=1$, the term $\left|0.5 \mathcal{E}\left\{\log _{2}\left(\eta_{1} \eta_{2}\right)\right\}\right|$ is always lower ${ }^{2}$ than $7 \cdot 10^{-3}$ for $0 \leq \chi<1$ : it can therefore be neglected with respect to the first term in (46), which is always equal to 1 . For low $\delta_{1}$ and $\delta_{2},(46)$ is therefore well approximated by

$$
\Delta \overline{\mathcal{I}} / n \approx 1+\log _{2}\left[\frac{1-e^{-4 / \xi_{t}}}{1-e^{-2 / \xi_{t}}}\right]+\log _{2}\left[\frac{1-e^{-4 / \xi_{r}}}{1-e^{-2 / \xi_{r}}}\right]
$$

2) Arbitrary SNR Analysis: At arbitrary SNR, the asymptotic mutual information of uni-polarized spatially correlated channels is well-known, and can be calculated using the Stieltjes transform [33]. Alternative methods can also be used (see [32] as an example). We eventually obtain that the asymptotic average mutual information per receive antenna $\overline{\mathcal{I}} / n$ is given by

$\frac{\overline{\mathcal{I}}}{n}=\frac{1}{n} \log _{2} \operatorname{det}\left(\mathbf{I}_{n}+\beta_{t} \boldsymbol{\Theta}_{r}\right)+\frac{1}{n} \log _{2} \operatorname{det}\left(\mathbf{I}_{n}+\beta_{r} \boldsymbol{\Theta}_{t}\right)-\frac{1}{\rho} \beta_{t} \beta_{r}$,

where $\beta_{t}$ and $\beta_{r}$ are the solutions of

$$
\left\{\begin{array}{l}
\beta_{t}=\frac{\rho}{n} \operatorname{Tr}\left[\boldsymbol{\Lambda}_{\Theta_{t}}\left(\mathbf{I}_{n}+\beta_{r} \boldsymbol{\Lambda}_{\Theta_{t}}\right)^{-1}\right] \\
\beta_{r}=\frac{\rho}{n} \operatorname{Tr}\left[\boldsymbol{\Lambda}_{\Theta_{r}}\left(\mathbf{I}_{n}+\beta_{t} \boldsymbol{\Lambda}_{\Theta_{r}}\right)^{-1}\right]
\end{array}\right.
$$

and $\boldsymbol{\Lambda}_{\Theta_{t}}$ and $\boldsymbol{\Lambda}_{\Theta_{r}}$ are diagonal matrices containing the eigenvalues of $\boldsymbol{\Theta}_{t}$ and $\boldsymbol{\Theta}_{r}$. Both correlation matrices have the form

$$
\boldsymbol{\Theta}=\left(\begin{array}{cccc}
1 & \varpi & \ldots & \varpi^{n-1} \\
\varpi & 1 & \ldots & \varpi^{n-2} \\
\vdots & \vdots & \ddots & \vdots \\
\varpi^{n-1} & \varpi^{n-2} & \ldots & 1
\end{array}\right),
$$

and it is known (see [34, p. 38]) that the eigenvalues of $\Theta$ converge uniformly (as $n \rightarrow \infty$ ) to

$$
\begin{aligned}
\lambda_{\Theta}(x) & =\sum_{k=0}^{\infty} \varpi^{k} e^{j k 2 \pi x}+\sum_{k=1}^{\infty} \varpi^{k} e^{-j k 2 \pi x} \\
& =\frac{1}{1-\varpi e^{j 2 \pi x}}+\frac{\varpi e^{-j 2 \pi x}}{1-\varpi e^{-j 2 \pi x}}
\end{aligned}
$$

for $x \in[0,1]$ and $\varpi=e^{-\frac{d_{t}}{\Delta_{t}}}$ or $e^{-\frac{d_{r}}{\Delta_{r}}}$. Therefore, the asymptotic ergodic mutual information per antenna is given

\footnotetext{
${ }^{2}$ Using Jensen's inequality to obtain an analytical upper-bound is not adequate here, as the upper-bound is very loose.
} 
by $\lim _{n \rightarrow \infty} \overline{\mathcal{I}} / n$

$$
\begin{aligned}
& =\int_{0}^{1} \log _{2}\left[1+\frac{\alpha_{t}}{1-e^{-\frac{d_{r}}{\Delta_{r}}} e^{j 2 \pi x}}+\frac{e^{-\frac{d_{r}}{\Delta_{r}}} \alpha_{t} e^{-j 2 \pi x}}{1-e^{-\frac{d_{r}}{\Delta_{r}}} e^{-j 2 \pi x}}\right] d x \\
& +\int_{0}^{1} \log _{2}\left[1+\frac{\alpha_{r}}{1-e^{-\frac{d_{t}}{\Delta_{t}}} e^{j 2 \pi x}}+\frac{e^{-\frac{d_{t}}{\Delta_{t}}} \alpha_{r} e^{-j 2 \pi x}}{1-e^{-\frac{d_{t}}{\Delta_{t}}} e^{-j 2 \pi x}}\right] d x \\
& -\frac{1}{\rho} \alpha_{t} \alpha_{r},
\end{aligned}
$$

where $\alpha_{t}$ and $\alpha_{r}$ are solutions of

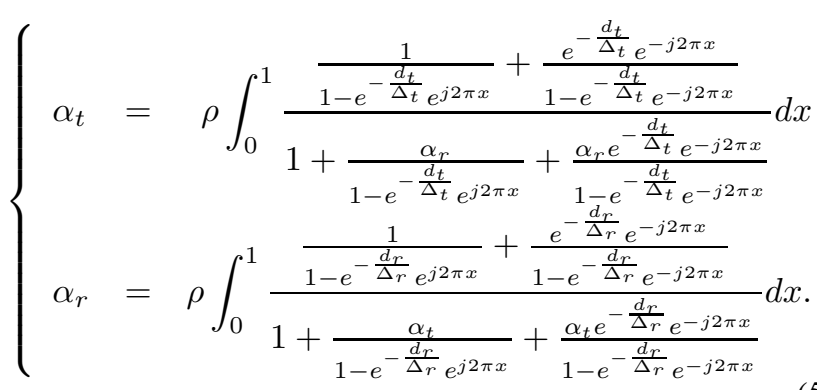

For dual-polarized schemes, assume first that the eigenvalues of $\tilde{\mathbf{X}} \tilde{\mathbf{X}}^{H}$ are fixed. In this case, the $n$ eigenvalues of $\tilde{\mathbf{H}}_{\times} \tilde{\mathbf{H}}_{\times}^{H}$ can be expressed as the product of the $n / 2$ eigenvalues of $\tilde{\mathbf{H}}^{\prime} \tilde{\mathbf{H}}^{\prime}{ }^{H}$ by $\eta_{1}$ and $\eta_{2}$ respectively. Hence, we may decompose the conditional ergodic mutual information per antenna as

$$
\begin{aligned}
\left.\frac{\overline{\mathcal{I}}_{\times}}{n}\right|_{\eta_{1}, \eta_{2}} & =\frac{1}{2} \int \log _{2}\left[1+\rho \eta_{1} \lambda\right] p_{\lambda}(\lambda) d \lambda \\
& +\frac{1}{2} \int \log _{2}\left[1+\rho \eta_{2} \lambda\right] p_{\lambda}(\lambda) d \lambda,
\end{aligned}
$$

where $\lambda$ designates the eigenvalues of $\tilde{\mathbf{H}}^{\prime} \tilde{\mathbf{H}}^{\prime}{ }^{H} / n$ and $p_{\lambda}(\lambda)$ is the limit probability density of $\lambda$ when $n \rightarrow \infty$. The latter can be quite easily evaluated, e.g. as described in [32]. When $\eta_{1}$ and $\eta_{2}$ are random, the quantities $\rho \eta_{1}$ and $\rho \eta_{2}$ can be thought of as randomly varying effective SNRs. The randomness is represented by the four phase-shifts $\phi_{k}, k=1, \ldots, 4$, which are uniformly distributed over $[0,2 \pi)$. The ergodic mutual information per antenna is finally given by

$$
\begin{aligned}
\frac{\overline{\mathcal{I}}_{\times}}{n} & =\frac{1}{32 \pi^{4}} \int_{0}^{2 \pi} \ldots \int_{0}^{2 \pi} \\
\sum_{k=1}^{2} & \left\{\int \log _{2}\left[1+\rho \eta_{k} \lambda\right] p_{\lambda}(\lambda) d \lambda\right\} d \phi_{1} \ldots d \phi_{4} .
\end{aligned}
$$

Simulation results are illustrated in Figure 2, which assumes that $\xi_{t}=\xi_{r}=\xi$ and that $\delta_{1}=\delta_{2}=0$. The minimum normalized antenna density $\xi_{\min }$ for which $\Delta \overline{\mathcal{I}} / n \geq 0$ is plotted for various values of $\chi$ (ranging from 0 to 0.9 ). The value of $\xi_{\text {min }}$ decreases as the SNR increases, and reaches its asymptotic value of 2.27 at high SNR. Note that the approximation of (47) is indeed well verified, in the sense that $\xi_{\min }$ is quasi-independent of $\chi$ at high SNR. The impact of $\chi$ is also pretty intuitive: for small XPR values, uni-polarized schemes remain attractive for larger densities, as the dualpolarized transmissions are heavily penalized by the energy loss, especially at low SNR levels. At low SNR, it is indeed

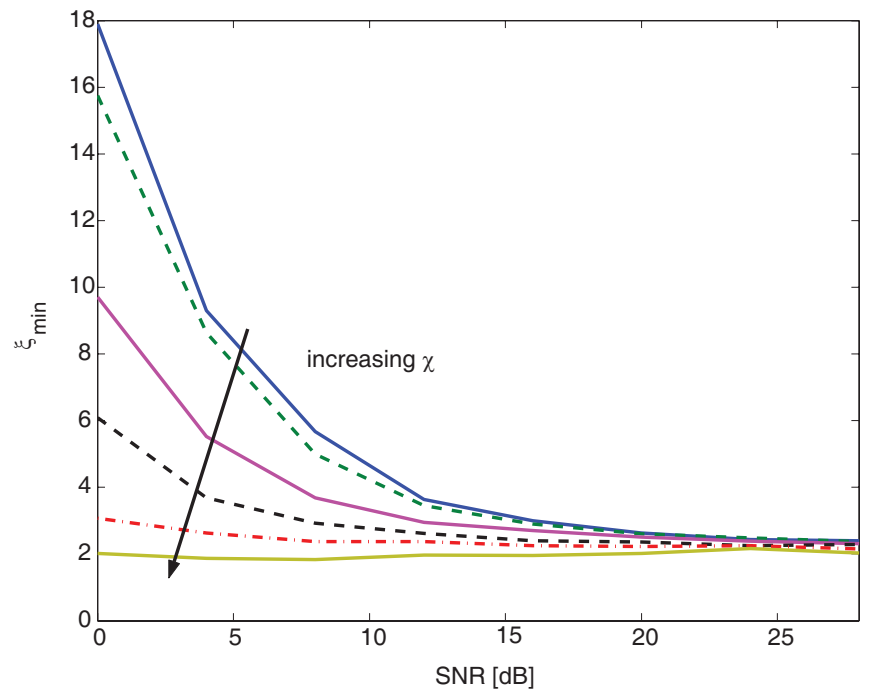

Fig. 2. Normalized antenna density $\xi_{\min }$ above which dual-polarization should be favored as a function of the SNR $\left(\chi^{-1}\right.$ is the channel XPR, $\chi \in$ $\{0,0.1,0.3,0.5,0.7,0.9\}$ and $\left.\delta_{1}=\delta_{2}=0\right)$.

well known that the mutual information is essentially linked to the channel energy [26]. Finally, note that the above discussion would yield the same values if one had considered the transmit SNR.

\section{Average Pairwise Error Probability in DUAL-POLARIZED TITO CHANNELS}

We are now interested in the average pairwise error probability (PEP) as a function of the SNR $\rho$, i.e. $P(\mathbf{C}, \mathbf{E})$ represents the probability that the receiver decodes the codeword $\mathbf{E}=\left[\mathbf{e}_{0} \ldots \mathbf{e}_{T-1}\right]$ instead of codeword $\mathbf{C}=\left[\mathbf{c}_{0} \ldots \mathbf{c}_{T-1}\right](T$ is the duration of the space-time block code). In $2 \times 2$ dualpolarized Rayleigh and LOS Ricean slow fading channels, the expressions of the PEP [35] are given by

$$
\begin{aligned}
& P(\mathbf{C}, \mathbf{E})=\frac{1}{\pi} \int_{0}^{\pi / 2} \\
& \quad \prod_{i=1}^{r\left(\mathbf{C}_{\mathbf{R}_{\times, \mathbf{a}}}\right)}\left(1+\Xi \lambda_{i}\left(\mathbf{C}_{\mathbf{R}_{\times, \mathbf{a}}}\right)\right)^{-1} \exp \left[-\Xi K \operatorname{vec}\left(\overline{\mathbf{H}}_{\times, a}^{H}\right)^{H}\right. \\
& \left.\quad\left(\mathbf{I}_{n_{r}} \otimes \ddot{\mathbf{E}}\right)\left(\mathbf{I}_{n_{r} n_{t}}+\Xi \mathbf{C}_{\mathbf{R}_{\times, \mathbf{a}}}\right)^{-1} \operatorname{vec}\left(\overline{\mathbf{H}}_{\times, a}^{H}\right)\right] d \beta
\end{aligned}
$$

where $\Xi=\rho /\left(4(1+K) \sin ^{2} \beta\right), \mathbf{C}_{\mathbf{R}_{\times, a}}=\mathbf{R}_{\times, a}\left(\mathbf{I}_{n_{r}} \otimes \ddot{\mathbf{E}}\right)$, $\mathbf{R}_{\times, a}$ is the correlation matrix of $\operatorname{vec}\left(\tilde{\mathbf{H}}_{\times, a}\right)$ and $\ddot{\mathbf{E}}=$ $(\mathbf{C}-\mathbf{E})(\mathbf{C}-\mathbf{E})^{H}$. In this paper, we restrict our analysis to two simple schemes:

- the Alamouti O-STBC $(T=2)$, which extends the principle of transmit/receive diversity,

- the Spatial Multiplexing scheme, which consists in sending different data streams over each antenna, thereby increasing the system throughput.

In Rayleigh fading channels with vertical and horizontal antennas at both ends, neglecting antenna XPI $\left(\chi_{a, t}=\chi_{a, r} \approx 0\right)$ and using the same models as those used when estimating the mutual information (see Section IV), we may write the dual- 
polarized correlation matrix as

$$
\mathbf{R}_{\times}=\left[\begin{array}{cccc}
1 & 0 & 0 & s_{1}^{*} \delta_{1}^{*} \sqrt{\mu} \\
0 & \mu \chi & s_{2}^{*} \delta_{2}^{*} \sqrt{\mu} \chi & 0 \\
0 & s_{2} \delta_{2} \sqrt{\mu} \chi & \chi & 0 \\
s_{1} \delta_{1} \sqrt{\mu} & 0 & 0 & \mu
\end{array}\right] .
$$

\section{A. Performance of Orthogonal Space-Time Block Coding}

Restricting our analysis to the Alamouti scheme, the codeword matrix is given by

$$
\mathbf{C}=\frac{1}{\sqrt{2}}\left[\begin{array}{cc}
c_{0} & -c_{1}^{*} \\
c_{1} & c_{0}^{*}
\end{array}\right]
$$

where $c_{0}$ and $c_{1}$ are two symbols of a given constellation, and we denote the codeword errors as $d_{0}=\left(c_{0}-e_{0}\right) / \sqrt{2}$ and $d_{1}=\left(c_{1}-e_{1}\right) / \sqrt{2}$.

1) Rayleigh Fading Channels: In Rayleigh fading channels, the matrix $\mathbf{C}_{\mathbf{R}_{(\times)}}$is rewritten for O-STBC as

$$
\mathbf{C}_{\mathbf{R}_{(\times)}}=\mathbf{R}_{(\times)}\left[\left|d_{0}\right|^{2}+\left|d_{1}\right|^{2}\right]
$$

where the notation $\mathbf{R}_{(\times)}$means that the relationship is valid for both $\mathbf{R}$ in (12) and $\mathbf{R}_{\times}$in (58). Therefore, the impact of the propagation channel on the error probability is directly given by the four eigenvalues of the correlation matrix. To minimize the average PEP, it is sufficient to maximize the following Chernoff bound [35],

$$
\operatorname{det}\left(\mathbf{I}_{4}+\frac{\rho}{4} \mathbf{C}_{\mathbf{R}_{(\times)}}\right)=\operatorname{det}\left(\mathbf{I}_{4}+\rho_{e} \mathbf{R}_{(\times)}\right)
$$

where we define for better legibility an effective SNR as $\rho_{e}=$ $\rho\left[\left|d_{0}\right|^{2}+\left|d_{1}\right|^{2}\right] / 4$. Dual-polarization is then preferred when

$$
\operatorname{det}\left(\mathbf{I}_{n_{r} n_{t}}+\rho_{e} \mathbf{R}_{\times}\right)>\operatorname{det}\left(\mathbf{I}_{n_{r} n_{t}}+\rho_{e} \mathbf{R}\right) .
$$

In the high SNR regime, the condition simply becomes

$$
\operatorname{det}\left(\mathbf{R}_{\times}\right)>\operatorname{det}(\mathbf{R}) \text {. }
$$

Note however that the SNR level for which one reaches the so-called high SNR regime depends on the eigenvalues of $\mathbf{R}_{\times}$. Hence, the high SNR regime assumption might not be realistic when $\chi$ and/or $\mu$ are small, i.e. when some eigenvalues of $\mathbf{R}_{\times}$are small. The above conditions are easily expressed in terms of the correlation coefficients, as well as $\mu$ and $\chi$. As an example, let us consider a Kronecker-structured channel uncorrelated at the receiver (hence, $r=s_{1}=s_{2}=0$ ). When $\mu=1$ (i.e. when co-polarized waves are equally attenuated), the above conditions are respectively rewritten as

$$
\begin{aligned}
& \left(1+\rho_{e}\right)^{2}\left(1+\chi \rho_{e}\right)^{2}> \\
& \left(1+\rho_{e}\right)^{4}+\rho_{e}^{2}|t|^{2}\left[\rho_{e}^{2}|t|^{2}-2\left(1+\rho_{e}\right)^{2}\right]
\end{aligned}
$$

and

$$
|t|>\sqrt{1-\chi^{2}}
$$

A similar condition on $|r|$ would be found for Kroneckerstructured channels uncorrelated at the transmitter. This indicates that dual-polarization can increase the performance at high spatial transmit/receive correlation levels, despite the reduction of the average energy of the channel caused by $\chi$. Naturally, the higher $\chi$, the lower the transmit/receive correlation for which dual-polarized schemes outperform unipolarized schemes. Note also that transmit and receive correlations equally affect the performance of diversity schemes. It might also seem sensible to use the effective diversity order $N_{d i v}$ as a natural metric to decide whether dual-polarized systems should be preferred to uni-polarized systems [36]. Defining

$$
N_{d i v}=\left[\frac{\operatorname{Tr}\{\mathbf{R}\}}{\|\mathbf{R}\|_{F}}\right]^{2},
$$

the condition under which dual-polarized transmissions (with same polarization scheme at both Tx and Rx) perform better than uni-polarized transmissions is given

- if $\delta_{1}=1$ and $\left|\delta_{2}\right|=1$ by

$$
\begin{array}{r}
\frac{(1+\mu+\mu \chi+\chi)^{2}}{1+\mu^{2}+\mu^{2} \chi^{2}+\chi^{2}+2 \mu\left(\left|s_{1}\right|^{2}+\chi^{2}\left|s_{2}\right|^{2}\right)}> \\
\frac{4}{1+|t|^{2}+|r|^{2}+\frac{\left|s_{1}\right|^{2}+\left|s_{2}\right|^{2}}{2}},
\end{array}
$$

- if $\delta_{1}=\delta_{2}=0$ by

$$
\frac{(1+\mu+\mu \chi+\chi)^{2}}{1+\mu^{2}+\mu^{2} \chi^{2}+\chi^{2}}>\frac{4}{1+|t|^{2}+|r|^{2}+\frac{\left|s_{1}\right|^{2}+\left|s_{2}\right|^{2}}{2}} .
$$

If we consider the same example as above $\left(r=s_{1}=s_{2}=0\right.$ and $\mu=1)$, (66) and (67) are both rewritten as

$$
|t|>\frac{1-\chi}{1+\chi}
$$

This condition is actually looser than (64) with respect to favoring dual-polarized transmissions, as it only implies that the number of degrees of freedom offered by dual-polarized channels be higher than that offered by uni-polarized channels. It does not imply that the error probabililty is lower. When $\frac{1-\chi}{1+\chi}<|t| \leq \sqrt{1-\chi^{2}}$, dual-polarized schemes exhibit a larger diversity order, but are still penalized by the energy loss since $\operatorname{Tr}\left\{\mathbf{R}_{\times}\right\}<\operatorname{Tr}\{\mathbf{R}\}$.

2) Ricean Fading Channels: We analyze this case assuming that the Ricean component is the LOS, that the K-factor is high (so that the Rayleigh component can be neglected) and that the transmit antenna XPI is large enough to be neglected. The performance solely depends on the Frobenius norm of the coherent component. Naturally, this norm is always smaller for dual-polarized schemes (since $\chi_{a, r} \leq 1$ ). The same conclusion is reached if we consider the effective diversity order (neglecting the Rayleigh component): $N_{d i v}=2 \mathrm{~K}$ for uni-polarized transmissions, while $N_{d i v}=\left(2+\chi_{a, r}\right) K / 2$ for dual-polarized systems. Therefore, the use of dual-polarized transmissions is not recommended for diversity schemes in Ricean channels.

\section{B. Performance of Spatial Multiplexing}

For a simple uncoded spatial multiplexing scheme, we have

$$
(\mathbf{C}-\mathbf{E})(\mathbf{C}-\mathbf{E})^{H}=\left[\begin{array}{ll}
\left|d_{0}^{2}\right| & d_{0} d_{1}^{*} \\
d_{0}^{*} d_{1} & \left|d_{1}\right|^{2}
\end{array}\right]
$$

1) Rayleigh Fading Channels: Given (69), the rank of both $\mathbf{C}_{\mathbf{R}}$ and $\mathbf{C}_{\mathbf{R} \times}$ is two, with their two non-zero eigenvalues $\lambda_{1,2}$ 


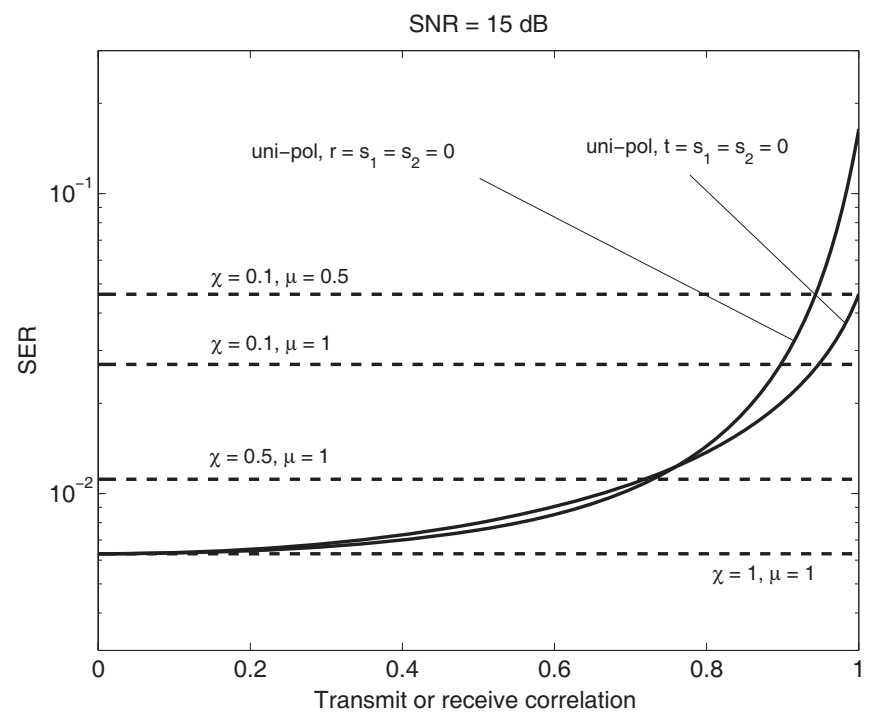

Fig. 3. Performance of QPSK Spatial Multiplexing for uni- (solid lines) and dual-polarized (dashed lines) transmissions as a function of $t$ or $r$.

reading as

$$
\lambda_{1,2}=\frac{a+d \pm \sqrt{(a-d)^{2}+4|b|^{2}}}{2}
$$

with

- for uni-polarized schemes

$$
\begin{aligned}
a & =\left|d_{0}\right|^{2}+\left|d_{1}\right|^{2}+2 \Re\left[t d_{0} d_{1}^{*}\right] \\
b & =r\left(\left|d_{0}\right|^{2}+\left|d_{1}\right|^{2}\right)+s_{1} d_{0} d_{1}^{*}+s_{2} d_{0}^{*} d_{1} \\
d & =a,
\end{aligned}
$$

- for dual-polarized schemes

$$
\begin{aligned}
a & =\left|d_{0}\right|^{2}+\mu \chi\left|d_{1}\right|^{2} \\
b & =\sqrt{\mu}\left(s_{1} \delta_{1} d_{0} d_{1}^{*}+s_{2} \delta_{2} \chi d_{0}^{*} d_{1}\right) \\
d & =\chi\left|d_{0}\right|^{2}+\mu\left|d_{1}\right|^{2} .
\end{aligned}
$$

An estimate of the average symbol error rate may be easily obtained by weighting the average PEP over the different possible symbols, using a union bound approximation. Various simulation results are illustrated in Figure 3 for a QPSK modulation, as a function of $t$ (assuming that $r=s_{1}=s_{2}=0$ ) or as a function of $r$ (assuming that $t=s_{1}=s_{2}=0$ ), for a constant receive SNR $\rho=15 \mathrm{~dB}$. Note that the comparison between various dual-polarized channels should be made with care, as a fixed transmit power constraint would vary the receive SNR. Yet, the comparison between each uni-polarized channel and each dual-polarized channel does not depend on the power constraint, as the channel depolarization is constant for each comparison.

Considering first uni-polarized systems (solid lines), we observe that transmit correlation is more harmful than receive correlation, as already noticed in [37]. As for the use of dual-polarized arrays (dashed lines), it is clear that these are only beneficial with respect to uni-polarized schemes when the transmit and/or receive correlations are higher than a certain level. This level is directly related to the CPR and XPR. The smaller $\mu$ and/or $\chi$, the larger this correlation level.

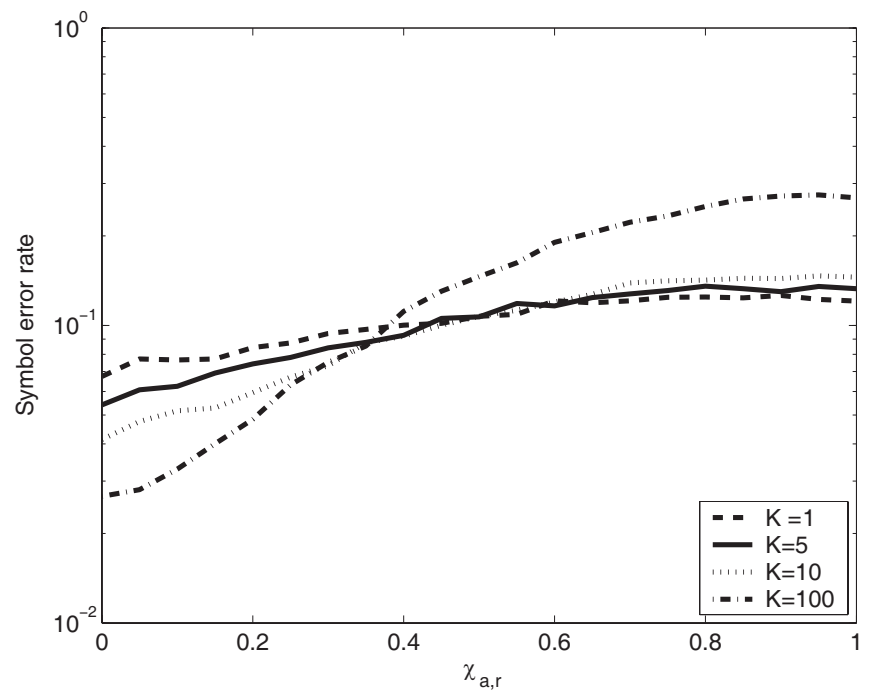

Fig. 4. Performance of QPSK Spatial Multiplexing in various Ricean channels as a function of Rx antenna XPI for a transmit SNR $\rho_{0}=10 \mathrm{~dB}$.

Naturally, when both $\mu$ and $\chi$ are equal to one, dual-polarized systems always perform better than uni-polarized systems, as the decorrelation effect is not hampered by a decrease of power. For $\mu$ and/or $\chi$ smaller than one, the required correlation increases rapidly. As a consequence, dual-polarized spatial multiplexing schemes perform well in Rayleigh fading channels when the uni-polarized spatial correlations are high enough.

2) Ricean Fading Channels: In LOS Ricean fading channels, the error probability is obtained through (57). Simulation results are displayed in Figure 4 for $\chi=0.1, \mu=1$, $\delta_{1}=\delta_{2}=0$ and $\rho_{0}=10 \mathrm{~dB}$. The cases of uni-polarized transmissions approximately correspond here to $\chi_{a, r}=1$ (the higher the K-factor, the better the approximation). Clearly, the use of dual-polarized antennas with large XPI significantly increases the performance, even when considering a constant transmit power.

\section{CONCLUSIONS}

In this paper, we have presented a simple analytical model of dual-polarized TITO and MIMO transmissions. The channel model efficiently separates the spatial and polarization effects, which makes it particularly easy to parametrize, thanks to a thorough overview of the literature, whose conclusions are summarized at the end of Section II.B.

As far as the benefits from dual-polarized arrays are concerned, the derivations point out that large multiplexing (i.e. capacity) gains are achievable in Ricean or highly correlated Rayleigh fading channels, but that diversity gains are only possible in highly correlated Rayleigh fading channels (and not in Ricean channels). Furthermore, our derivations have highlighted that there is a fundamental trade-off between the array density and the capacity gain, i.e. for a given array geometrical extension, there is a critical antenna density above which dual-polarized arrays should be preferred. Finally, our analysis identifies quantitatively for which channels (correlations, K-factor), schemes (O-SBTC or Spatial Multiplexing) 
and SNR levels the use of multiple polarizations might become beneficial.

\section{REFERENCES}

[1] T. Neubauer and P. Eggers, "Simultaneous characterization of polarization matrix components in pico cells," in Proc. VTC-F 1999 - IEEE Vehicular Technology Conf. Fall, 1999, pp. 1361-1365.

[2] T. Svantesson, "A double-bounce channel model for multi-polarized MIMO systems," in Proc. VTC-F 2002 - IEEE Vehicular Technology Conf. Fall, 2002, pp. 691-695.

[3] P. Soma, D. Baum, V. Erceg, R. Krishnamoorthy, and A. Paulraj, "Analysis and modeling of multiple-input multiple-output radio channels based on outdoor measurements conducted at $2.5 \mathrm{GHz}$ for fixed BWA applications," in Proc. ICC 2002 - IEEE Int. Conf. Commun., vol. 1, New York, NY, May 2002, pp. 272-276.

[4] C. Oestges, V. Erceg, and A. Paulraj, "Propagation modeling of multipolarized MIMO fixed wireless channels," IEEE Trans. Veh. Technol., vol. 53, no. 3, pp. 644-654, May 2004.

[5] M. Shafi, M. Zhang, A. Moustakas, P. Smith, A. Molisch, F. Tufvesson, and S. Simon, "Polarized MIMO channels in 3-D: models, measurements and mutual information," IEEE J. Select. Areas Commun., vol. 24 no. 3, pp. 514-527, Mar. 2006.

[6] G. Calcev, D. Chizhik, B. Goransson, S. Howard, H. Huang, A. Kogiantis, A. Molisch, A. Moustakas, D. Reed, and H. Xu, "A wideband spatial channel model for system-wide simulations," IEEE Trans. Veh. Technol., vol. 56, no. 2, pp. 389-403, Mar. 2007.

[7] W. Kotterman, G. Sommerkorn, and R. Thomä, "Cross-correlation values for dual-polarised indoor MIMO links and realistic antenna elements," in Proc. 3rd Int. Symp. Wireless Communication Systems, Valencia, Spain, Sept. 2006

[8] W. Kotterman, M. Landmann, G. Sommerkorn, and R. Thomä, "Power ratios and distributions in indoor NLOS channels for dual-polarized $2 \mathrm{x}$ 2 MIMO systems," in Proc. NEWCOM-ACoRN Joint Workshop, Vienna, Austria, Sept. 2006.

[9] H. Asplund, J. Berg, J. Medbo, and M. Riback, "Propagation characteristics of polarized radio waves in cellular communications," in Proc. VTC 2007 Fall - IEEE 66th Vehicular Technology Conf., Baltimore, MD, Sept. 2007.

[10] D. Shiu, G. Foschini, M. Gans, and J. Kahn, "Fading correlation and its effect on the capacity of multielement antenna systems," IEEE Trans. Commun., vol. 48, no. 3, pp. 502-513, Mar. 2000.

[11] C. Chuah, D. Tse, J. Kahn, and R. Valenzuela, "Capacity scaling in MIMO wireless systems under correlated fading," IEEE Trans. Inform. Theory, vol. 48, no. 3, pp. 637-650, Mar. 2002.

[12] W. Weichselberger, M. Herdin, H. Özcelik, and E. Bonek, "A stochastic MIMO channel model with joint correlation of both link ends," IEEE Trans. Wireless Commun., to be published.

[13] S. Kozono, T. Tsuruhara, and M. Sakamoto, "Base station polarization diversity reception for mobile radio," IEEE Trans. Veh. Technol., vol. 33, no. 4, pp. 301-306, Apr. 1984.

[14] R. Vaughan, "Polarization diversity in mobile communications," IEEE Trans. Veh. Technol., vol. 39, no. 3, pp. 177-186, Mar. 1990.

[15] J. Lempainen and J. Laiho-Steffens, "The performance of polarization diversity schemes at a base station in small/micro cells at $1800 \mathrm{MHz}$," IEEE Trans. Veh. Technol., vol. 47, no. 3, pp. 1087-1092, Mar. 1998.

[16] F. Lotse, J. Berg, et al., "Base station polarization diversity reception in macrocellular systems at $1800 \mathrm{MHz}$," in Proc. VTC 1996 - IEEE 46th Vehicular Technology Conf., 1996, pp. 1643-1646.

[17] T. Sorensen, A. Nielsen, P. Mogensen, M. Tolstrup, and K. Steffensen, "Performance of two-branch polarisation antenna diversity in an operational GSM network," in Proc. VTC 1998 - IEEE 48th Vehicular Technology Conf., 1998, pp. 741-746.

[18] A. Kainulainen, L. Vuokko, and P. Vainikainen, "Polarization behavior in different urban radio environments at $5.3 \mathrm{GHz}$," COST 273, Tech. Rep. 05-018, Jan. 2005.

[19] D. Chizhik, J. Ling, D. Samardzija, and R. Valenzuela, "Spatial and polarization characterization of MIMO channels in rural environment," in Proc. VTC 2005 Spring - IEEE 61st Vehicular Technology Conf., vol. 1, Stockholm, Sweden, May 2005, pp. 161-164.

[20] P. Kyritsi, D. Cox, R. Valenzuela, and P. Wolniansky, "Correlation analysis based on MIMO channel measurements in an indoor environment," IEEE J. Select. Areas Commun., vol. 21, no. 5, pp. 713-720, June 2003.

[21] J. Wallace and M. Jensen, "Modeling the indoor MIMO wireless channel," IEEE Trans. Antennas Propagat., vol. 50, no. 5, p. 591-599, May 2002.
[22] J. Hämäläinen, R. Wichman, J. Nuutinen, J. Ylitalo, and T. Jamsa, "Analysis and measurements for indoor polarization MIMO in $5.25 \mathrm{GHz}$ band," in Proc. VTC 2005 Spring - IEEE 61st Vehicular Technology Conf., vol. 1, Stockholm, Sweden, May 2005, pp. 252-256.

[23] C. Oestges, B. Clerckx, L. Raynaud, and D. Vanhoenacker-Janvier, "Deterministic channel modeling and performance simulation of microcellular wideband communication systems," IEEE Trans. Veh. Technol., vol. 51, no. 6, pp. 1422-1430, June 2002.

[24] B. Lee, S. Kwon, H. Moon, J. Lim, J. Seok, C. Mun, and Y. Yoon, "Modeling the indoor channel for the MIMO system using dual polarization antennas," in Peoc. 9th European Conference on Wireless Technology, Manchester, UK, Sept. 2006.

[25] L. Correia, COST 259 - Wireless flexible personalized communications. London: Wiley, 2001.

[26] A. Paulraj, R. Nabar, and D. Gore, Introduction to Space-Time Wireless Communications. Cambridge, UK: Cambridge University Press, 2003.

[27] C. Oestges and A. Paulraj, "Beneficial impact of channel correlations on MIMO capacity," Electron. Lett., vol. 40, no. 10, pp. 606-607, May 2004.

[28] M. Coldrey, "Modeling and capacity of polarized MIMO channels," in Proc. VTC 2008 Spring - IEEE 67th Vehicular Technology Conf.

[29] A. Lozano, A. Tulino, and S. Verdú, "Multiple-antenna capacity in the low-power regime," IEEE Trans. Inform. Theory, vol. 49, no. 10, pp. 2527-2544, Oct. 2003.

[30] M. Hunukumbure and M. Beach, "MIMO channel measurements and analysis with prototype user devices in a $2 \mathrm{GHz}$ urban cell," in Proc. PIMRC 2006 - IEEE 17th Int. Symp. on Pers., Indoor and Mobile Radio Commun., Helsinki, Finland, Sept. 2006.

[31] H. Özcelik and C. Oestges, "Some remarkable properties of diagonally correlated MIMO channels," IEEE Trans. Veh. Technol., vol. 54, no. 6 , pp. 2143-2145, Nov. 2005

[32] C. Martin and B. Ottersten, "Asymptotic eigenvalue distributions and capacity for MIMO channels under correlated fading," IEEE Trans. Wireless Commun., vol. 3, no. 4, pp. 1350-1359, July 2004.

[33] J. Dumont, P. Loubaton, S. Lasaulce, and M. Debbah, "On the asymptotic performance of MIMO correlated Ricean channels," in Proc. ICASSP 2005 - IEEE Int. Conf. Acoust. Speech and Signal Processing, Philadelphia, PA, Mar. 2005, pp. 813-816.

[34] R. Gray, "Toeplitz and circulant matrices: a review," Foundations and Trends in Commun. and Inform. Theory, vol. 2, no. 3, pp. 155-239, 2006.

[35] C. Oestges and B. Clerckx, MIMO Wireless Communications: From Real-World Propagation to Space-Time Code Design. London: Academic Press, 2007.

[36] R. Nabar, "Performance analysis and transmit optimization for general MIMO channels," Ph.D. dissertation, Stanford University, Feb. 2003.

[37] R. Nabar, H. Boelcskei, V. Erceg, D. Gesbert, and A. Paulraj, "Performance of multiantenna signaling techniques in the presence of polarization diversity," IEEE Trans. Signal Processing, vol. 50, no. 10, pp. 2553-2562, Oct. 2002.

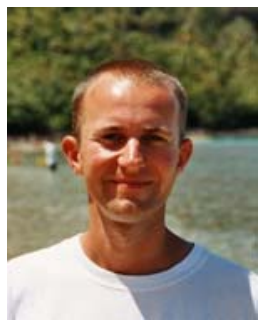

Claude Oestges received the M.Sc. and Ph.D. degrees in Electrical Engineering from the Université catholique de Louvain (UCL), Louvain-la-Neuve, Belgium, respectively in 1996 and 2000. In January 2001, he joined as a post-doctoral scholar the Smart Antennas Research Group (Information Systems Laboratory), Stanford University, CA, USA. From January 2002 to September 2005, he was associated with the Microwave Laboratory UCL as a postdoctoral fellow of the Belgian Fonds de la Recherche Scientifique (FRS). He is presently a FRS Research Associate and Associate Professor at UCL. Claude Oestges has received the 1999-2000 IEE Marconi Premium Award and the 2004 IEEE Vehicular Technology Society Neal Shepherd Award. He is the author or co-author of one book and more than 70 research papers and communications. 


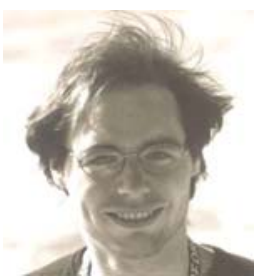

Bruno Clerckx received the M.Sc. and Ph.D. degrees in Electrical Engineering from the Université catholique de Louvain (UCL), Louvain-la-Neuve, Belgium, respectively in 2000 and 2005 . From September 2000 to September 2005, he was with the Microwave Laboratory UCL, first as a Research Assistant (until September 2001), then as a $\mathrm{PhD}$ student funded by the Belgian fund FRIA. From January 2003 to August 2003, he held a visiting research position at the Smart Antennas Research Group (Information Systems Laboratory), Stanford University, CA, USA, while from October 2004 to February 2005, he visited the Mobile Communication Department of Eurecom Institute, SophiaAntipolis, France. He is currently with the Communication and Networking Laboratory, Samsung Advanced Institute of Technology (SAIT), Korea. Dr. Clerckx is the author or co-author of one book and more than 30 research papers and communications and received the IEEE Symposium on Communications and Vehicular Technology Best Student Paper Award in 2002.

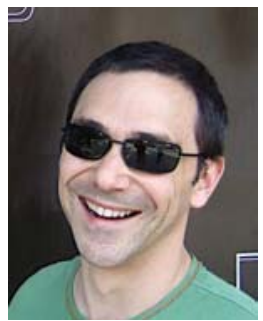

Maxime Guillaud received the M.Sc. degree in Electronics Engineering from Ecole Nationale Supérieure de l'Electronique et de ses Applications (ENSEA), Cergy, France, in 2000, and the Ph.D. in Electrical Engineering and Communications from Ecole Nationale Supérieure des Télécommunications (ENST), Paris, France, in 2005. From 2000 to 2001, he was a research engineer at Lucent Technologies' Bell Laboratories in Holmdel, NJ, USA. From 2005 to 2006 he was a post-doctoral researcher at Institut Eurecom, in Sophia-Antipolis, France. Since 2006, he is a Senior Researcher at the FTW telecommunications research center, Vienna, Austria. Dr. Guillaud is the author of more that 25 research papers and communications, and the recipient of a SPAWC 2005 student paper award. He worked on the transceiver architecture of cellular systems, as well as on various aspects of wireless channel modeling, including sparse representations and maximum entropy inference. He introduced the principle of relative calibration for the exploitation of channel reciprocity. His current research topics revolve around coordination and cooperation in multi-user wireless communications.

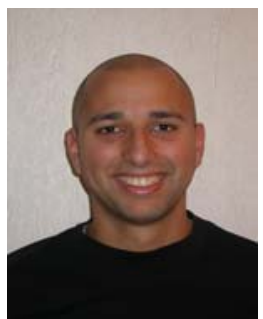

Mérouane Debbah was born in Madrid, Spain. He entered the Ecole Normale Supérieure de Cachan (France) in 1996 where he received the M.Sc. and the Ph.D. degrees respectively in 1999 and 2002. From 1999 to 2002, he worked for Motorola Labs on Wireless Local Area Networks and prospective fourth generation systems. From 2002 until 2003, he was appointed Senior Researcher at the Vienna Research Center for Telecommunications (ftw.), Vienna, Austria working on MIMO wireless channel modeling issues. From 2003 until 2007, he joined the Mobile Communications department of the Institute Eurecom (Sophia Antipolis, France) as an Assistant Professor. He is presently a Professor at Supelec (Gif-sur-Yvette, France), holder of the Alcatel-Lucent Chair on flexible radio. His research interests are in information theory, signal processing and wireless communications. 